Chapter 7

\title{
Advances in Infrared Detector Array Technology
}

\author{
Nibir K. Dhar, Ravi Dat and Ashok K. Sood \\ Additional information is available at the end of the chapter \\ http://dx.doi.org/10.5772/51665
}

\section{Introduction}

This Chapter covers recent advances in Short Wavelength Infrared (SWIR), Medium Wavelength Infrared (MWIR) and Long Wavelength Infrared (LWIR) materials and device technologies for a variety of defense and commercial applications. Infrared technology is critical for military and security applications, as well as increasingly being used in many commercial products such as medical diagnostics, drivers' enhanced vision, machine vision and a multitude of other applications, including consumer products. The key enablers of such infrared products are the detector materials and designs used to fabricate focal plane arrays (FPAs).

Since the 1950s, there has been considerable progress towards the materials development and device design innovations. In particular, significant advances have been made during the past decade in the band-gap engineering of various compound semiconductors that has led to new and emerging detector architectures. Advances in optoelectronics related materials science, such as metamaterials and nanostructures, have opened doors for new approaches to apply device design methodologies, which are expected to offer enhanced performance and low cost products in a wide range of applications.

This chapter reviews advancements in the mainstream detector technologies and presents different device architectures and discussions. The chapter introduces the basics of infrared detection physics and various infrared wavelength band characteristics. The subject is divided into individual infrared atmospheric transmission windows to address related materials, detector design and device performance. Advances in pixel scaling, junction formation, materials growth, and processing technologies are discussed.

We discuss the SWIR band (1-3 microns) and address some of the recent advances in InGaAs, SiGe and HgCdTe based technologies and their applications. We also discuss MWIR band that covers 3-5 microns, and its applications. Some of the key work discussed includes InSb, HgCdTe, and III-V based Strained Layer Super Lattice (SLS) and barrier detector tech- 
nologies (nBn). Each of these technologies has a place in the IR applications where a variety of detector configurations can be used.

We also present a discussion on the LWIR band that covers the wavelength range between 8 and 14 microns. The technologies that are addressed are bolometer (Microbolometer Arrays), $\mathrm{HgCdTe}$ arrays, and a variety of very ingenious band-gap engineered devices using III-V compound semiconductor materials.

\section{Infrared Spectrum and Bands of Interest}

The word "infrared" refers to a broad portion of the electromagnetic spectrum that spans a wavelength range from $1.0 \mathrm{um}$ to beyond 30 um everything between visible light and microwave radiation. Much of the infrared spectrum is not useful for ground- or sea-based imaging because the radiation is blocked by the atmosphere. The remaining portions of the spectrum are often called "atmospheric transmission windows," and define the infrared bands that are usable on Earth. The infrared spectrum is loosely segmented into near infrared (NIR, 0.8-1.1um), short wave infrared (SWIR, 0.9-2.5um), mid wave infrared (MWIR, 3-5um), long wave infrared (LWIR, 8-14um), very long wave infrared (VLWIR, 12- 25um) and far infrared (FIR, > 25um), as shown in Figure 1. The MWIR- LWIR wavebands are important for the imaging of objects that emit thermal radiation, while the NIR-SWIR bands are good for imaging scenes that reflect light, similar to visible light. Some of the materials technologies and device architectures used for detector fabrication in the various IR bands are discussed in references $1-4$. Since NIR and SWIR are so near to the visible bands, their behavior is similar to the more familiar visible light. Energy in these bands must be reflected from the scene in order to produce good imagery, which means that there must be some external illumination source. Both NIR and SWIR imaging systems can take advantage of sunlight, moonlight, starlight, and an atmospheric phenomenon called "nightglow," but typically require some type of artificial illumination at night. In lieu of photon starved scenes, arrays of infrared Light Emitting Diodes (LEDs) can provide a very cost effective solution for short-range illumination. However, achieving good performance at distances of over hundreds of meters requires more directed illumination, such as a focused beam from a laser or specialized spotlight, although special consideration of eye-safety issues is required.

NIR and SWIR imaging systems often employ sensors that are more exotic than those found in consumer-grade camcorders and digital cameras. Because NIR has a wavelength longer than visible light, and SWIR a wavelength that is longer still, energy in these bands is scattered less by particles suspended in the atmosphere. Consequently, SWIR, and to a lesser extent NIR, systems are tolerant of low levels of obscurants like fog and smoke compared to visible light.

An imaging system that operates in the MWIR and LWIR ranges can be completely passive, requiring no external illumination, because the thermal imager is able to sense the energy that is emitted directly from objects in the scene. The major factors that determine how bright an object appears to a thermal imager are: the object's temperature and its emissivity. As an object gets hotter, it radiates more energy and appear brighter to a thermal imaging 
system. Emissivity is a physical property of materials that describes how efficiently it radiates heat. Because cloth has a lower emissivity than skin, the former will appear darker in a thermal imager even when both are exactly at the same temperature.

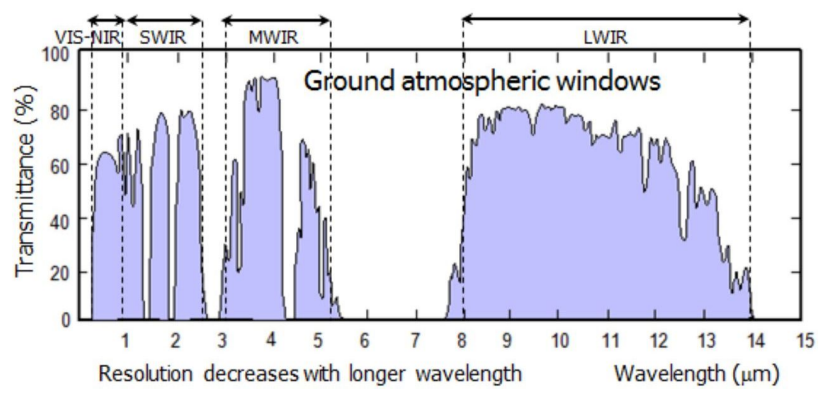

Figure 1. Definition of IR Spectral Band.

At the MWIR and LWIR wavelengths, infrared radiation behaves differently from visible light. For example, glass is transparent to wavelengths less than $3.0 \mu \mathrm{m}$, so glass optics can be used and windows can be seen through at these wavelengths. However, glass is opaque in the LWIR band and blocks most energy in the MWIR band. Consequently, the optics in LWIR and MWIR imaging systems cannot use inexpensive glass lenses, but are forced to use more expensive materials, such as germanium. Because glass windows are not transparent at the longer wavebands, they can appear to be brighter or darker according to their temperatures. Another difficulty with radiation in the MWIR and LWIR bands is that it is not transmitted through water. Imaging of a water (rain) coated scene with MWIR-LWIR wavelengths can wash out much of the scene's thermal contrast, resulting in a duller image.

The choice of wavelength band to exploit for IR imaging depends on the type of atmospheric conditions/obscurants between the target and the imager. Generally, atmospheric obscurants, such as haze or conventional smoke, cause much less scattering in the MWIR and LWIR bands than in the VIS-NIR or SWIR bands. This is because the haze or smoke particle size $(\sim 0.5 \mathrm{um})$ is much smaller than the IR wavelength (Rayleigh scattering). Obscurants such as fog and clouds can cause more scattering, since the particle size is comparable with the IR wavelength (Mie scattering). Infrared cameras sensitive to the longer wavelengths are more tolerant to smoke, dust and fog. In addition to obscurants, atmospheric turbulence can dictate the choice of IR wave band for a given application. The effects of optical turbulence, due to the fluctuations in the refractive index of the atmosphere, can add up over very long distances to impact range performance (blurring and image motion), allowing LWIR an edge over MWIR. As a rule of thumb, longer the wavelength better is the transmission through the earth's atmosphere.

According to Wien's Law, hotter objects emit more of their energy at shorter wavelengths. A blackbody source at $300 \mathrm{~K}$ has a peak exitance (power per unit area leaving a surface) at a wavelength of about $9.7 \mu \mathrm{m}$. For a source at $1000 \mathrm{~K}$, the maximum exitance occurs at $2.9 \mu \mathrm{m}$. Therefore, detectors operating in the LWIR band are well suited to image room temperature 
objects (people, buildings etc.), while MWIR band imagers are good for viewing objects at higher temperatures (hot engines and exhaust gasses). In general, LWIR and MWIR bands will produce thermal images if small temperature changes or varying emissivities exist within a scene. However, while the LWIR band imagery may exhibit a higher sensitivity for room temperature objects, the MWIR band imagery presents a better resolution.

\section{Theoretical Considerations}

IR detectors can be categorized as being either a quantum or thermal device. In a quantum detector, electromagnetic radiation absorbed in a semiconductor material generates electronhole pairs (EHP), which are sensed by an electronic readout circuit (ROIC). In a thermal detector, on the other hand, the incident IR photons are absorbed by a thermally isolated detector element, resulting in an increase in the temperature of the element. The temperature is sensed by monitoring an electrical parameter such as resistivity or capacitance.

Because thermal detectors depend on the quantity of heat absorbed, their response is independent of wavelength, however, the sensitivity depends on the material design for sensing. There are two types of quantum detectors: a) photoconductive (PC) where the electrical conductivity of the semiconductor changes as a function of the photon intensity; b) photovoltaic (PV) where a voltage is generated across a PN junction as photons impinge the semiconductor. Quantum detectors convert photons directly into charge carriers and no intermediate process is involved, such as the heating in a thermal detector to cause a change of a measurable electrical property.

Due to the various mechanisms used by detectors to convert optical to electrical signals, several figures of merit (FOM) are used to characterize their performance [5-8]. The output of the detector consists of its response signal to the incident radiation and random noise fluctuations. One such FOM is the Responsivity (R) of the detector, defined as the ratio of the root mean squared (rms) value of the signal voltage $\left(V_{s}\right)$ to the rms power (P in volts/watt) incident on the detector. The total power on an area $\left(A_{d}\right)$ is associated with an irradiance $\mathrm{E}$ (in watts $\mathrm{cm}^{2}$ ). Therefore,

$$
R=\frac{V_{s}}{P}=\frac{V_{s}}{E A_{d}}=[\text { volts } / \text { watts }]
$$

The random fluctuations in a detector's output limit its sensitivity to a certain minimum detectable power. The power necessary to generate an output signal equal to the noise is known as the Noise Equivalent Power (NEP). NEP is determined by measuring the amount of radiative power from a blackbody that falls on the detector to produce an rms signal $\mathrm{V}_{\mathrm{s}}$ equal to that generated by the detector noise $V_{N}$, when it is shuttered from the blackbody. NEP must be specified for a particular source temperature (T), modulation frequency (f), system bandwidth (BW), and detector area $\left(A_{d}\right)$. 


$$
N E P(T, f, B W)=\frac{P}{S / N}=\frac{E A_{d}}{S / N}=\frac{E A_{d}}{V_{s} / V_{N}}[\text { watts }]
$$

The inverse of NEP is referred to as the Detectivity:

$$
\text { Detectivity, } D=1 / \text { NEP }
$$

To have a meaningful comparison between different detectors, their respective performance must be reduced to representative conditions, so that the detectivity is often normalized to a bandwidth of $1 \mathrm{~Hz}$ and a detector area of $1 \mathrm{~cm}^{2}$. This figure of merit is called D-star $\left(\mathrm{D}^{*}\right)$ and can be interpreted as the signal to noise ration (SNR) out of a detector when 1 watt of radiant power is incident on a $1 \mathrm{~cm}^{2}$ detector area at a noise equivalent bandwidth of $1 \mathrm{~Hz}$.

$$
D^{*}=\frac{\sqrt{A_{d} B W}}{N E P}\left[\left.\mathrm{~cm} \sqrt{\mathrm{Hz}}\right|_{\text {watt }}\right]
$$

The performance of low-noise detectors may also be limited by radiative noise arriving at the detector from the background environment. When the background photon flux is much greater than the signal flux, the photodetector is said to be background-limited in performance or in the BLIP mode. The resulting detectivity of the photovoltaic detector is called $D_{B L I P}^{*}$ and is expressed as:

$$
D_{B L I P}^{*}=\frac{\lambda}{h c} \sqrt{\frac{\eta}{2 \phi_{B}}}
$$

where $\lambda$ is the wavelength, $\eta$ is the quantum efficiency, and $\phi_{B}$ is the incident photon flux in photons $/ \mathrm{cm}^{2}$-s. Equation (5) is valid for photovoltaic detectors which are shot-noise limited. For photoconductive detectors that are generation-recombination (G-R) noise limited, the $D_{B L I P}^{*}$ is lower by a factor of $\sqrt{2}$. The variances of the G-R noise are additive, causing an increase in the rms noise voltage by a factor of $\sqrt{2}$.

Background-limited performance of detectors can be improved by reducing the background photon flux, $\phi_{B}$. There are two ways of implementing this: a) use a cooled and/or spectral filter to limit the spectral band, or b) use a cold shield to limit the angular FOV of the detector. The former approach eliminates most of the background radiation from spectral regions in which the detector does not need to respond. The best detectors can approach background limited detectivities by limiting the field of view with a cold shield.Detectivity curves across the infrared spectrum for various commercially available detectors are shown in Figure 2 [7]. Calculated detectivities for the background-limited performance for ideal photon and thermal detectors are also included in Figure 2 as dashed curves. 


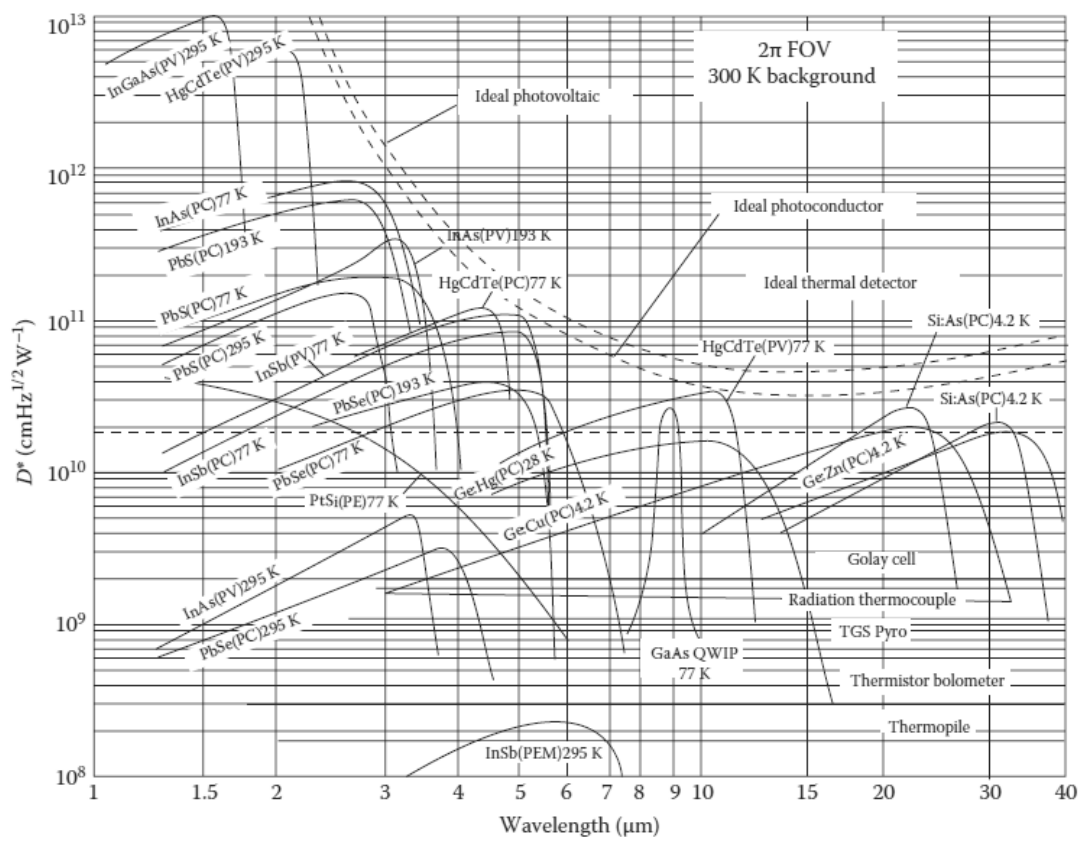

Figure 2. Detectivity curves for various commercially available photon and thermal IR detectors. Calculated detectivities are indicated by dashed lines [7].

Another frequently quoted figure of merit for a photodiode is its $R_{0} A$ product, where $R_{0}$ is the dynamic resistance of the photodiode and is equal to the slope of the I-V curve at the zero bias voltage point. This FOM is independent of the junction area, except when the dimensions are comparable to the minority carrier diffusion length.

Thermal detectors require a temperature change to produce a signal and do not generally need cooling, in contrast to photo detectors which are cooled to minimize noise. Absorbed radiation causes a temperature change that alters a temperature sensitive property of the detector which can be measured externally. A few examples include: electrical resistance in a bolometer, thermal expansion of Golay cells, and polarization in pyroelectric materials. Since these detectors depend on temperature changes resulting from incident radiation, they must be thermally isolated from their surroundings and have low thermal capacities for fast response to the radiation. In the case of a bolometer, the FOM is its thermal time constant which is defined as:

$$
\tau_{t h}=\frac{C_{t h}}{G_{t h}}=C_{t h} R_{t h}
$$

where $C_{t h}$ is the thermal capacity of the detector, $R_{t h}$ is the thermal resistance and $G_{t h}$ is the thermal coupling of the detector to its surroundings. The interaction of the bolometer with 
the incident radiation needs to be optimized while, simultaneously, minimizing its thermal contacts to the surroundings. In practice, this requires a bolometer with small mass and fine connecting wires to the heat sink.

\section{IR Material growth Techniques for HgCdTe}

$\mathrm{Hg}_{1-\mathrm{x}} \mathrm{Cd}_{\mathrm{x}} \mathrm{Te}$ (MCT) is the most widely used infrared (IR) detector material in military applications, compared to other IR detector materials, primarily because of two key features: it is a direct energy band gap semiconductor and its band gap can be engineered by varying the $\mathrm{Cd}$ composition to cover a broad range of wavelengths. The direct band gap of MCT allows for a high absorption of IR radiation, yielding high quantum efficiency in a relatively thin detector structure. As the $\mathrm{Cd}$ mole fraction, $\mathrm{x}$, increases, the energy gap for MCT increases linearly from a semimetal ( $\mathrm{HgTe}$ ) to a wide band gap semiconductor (CdTe).

The ability to tune the band gap of MCT enables IR detectors to operate in the wavelength bands ranging from SWIR to VLWIR (0.7-30 microns). For low-cost high-performance detectors, the MCT material must be produced on large diameter wafers with low defect densities and reproducible stoichiometric properties. These requirements are satisfied by a host of crystal growth techniques ranging from high temperature, melt grown bulk crystals, to low temperature, multilayer epitaxial layers.

Depending on the detector architecture, the crystal growth strategy could utilize any of the following techniques: Bulk Crystal Growth, Liquid Phase Epitaxy (LPE), Metal-organic Chemical Vapor Deposition (MOCVD), and Molecular Beam Epitaxy (MBE). The sections below will highlight each of these growth techniques with references to publications that will provide additional coverage.

\subsection{Bulk Crystal Growth}

Bulk crystal growth of MCT continues to play an important role in producing IR detector materials for photoconductive arrays, despite the progress made with various epitaxial thin film deposition techniques. Bulk growth process is typically used for large area single detectors for applications such as spectrometry. However, for photovoltaic arrays there are challenges associated with crystal grain boundaries, which are electrically active and contribute to line defects. Also there are limitations in the ingot diameter, which makes bulk growth suitable for only quad or single detector arrays. Several methods have been developed for growing MCT bulk crystals: Solid State Recrystallization (SSR), Traveling Heater Method (THM), Bridgman, Czochralski, Slush Growth, and Zone Melting [9-14]. This section will cover SSR and THM techniques.

The general challenge with melt grown MCT is to maintain a relatively high $\mathrm{Hg}$ vapor pressure during growth; otherwise, it is difficult to control the stoichiometry of the grown crystal. Also, the large separation between the liquidus and solidus compositions (see Figure 3) across a constant thermal tie line can result in a steady variation in the composition of a moving growth interface. 


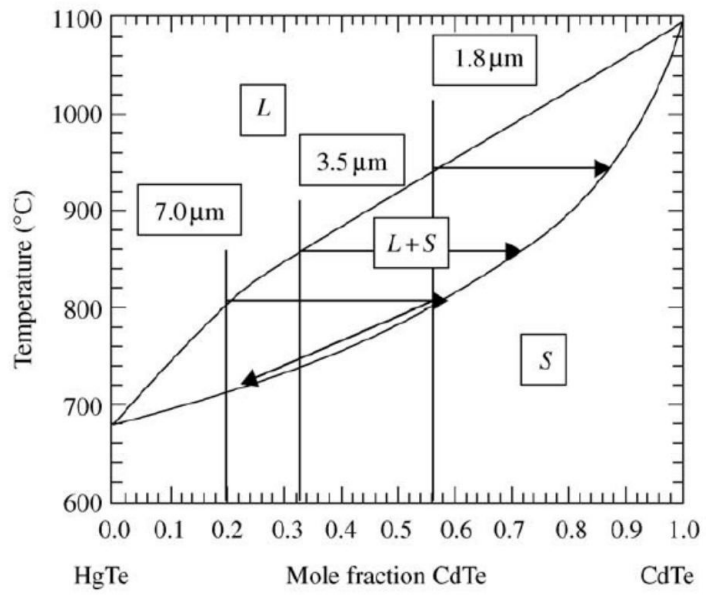

Figure 3. $T$ - x phase diagram for the pseudo-binary CdTe-HgTe [14].

\subsubsection{Solid State Recrystallization}

The SSR technique is used to alleviate the compositional variation at the growth interface in ternary systems, such as MCT, where the solidus and liquidus lines are widely separated. In the basic technique, the three high purity elements of MCT are cleaned and loaded into a thick walled, small diameter quartz ampoule that is evacuated, sealed and placed into a furnace. The ampoule is heated to approximately $950{ }^{\circ} \mathrm{C}$ and the melt is mixed by rocking the furnace. The MCT ampoule is removed from the furnace and rapidly quenched to produce a uniform composition.

The rapid quenching produces a dendritic structure in the MCT that is reduced by an extended (several days) recrystallization step, at temperatures just below the melting point. Grain growth occurs during the re-crystallization step and remaining compositional inhomogeneities are removed. On the down side, the high melt temperatures and very high $\mathrm{Hg}$ vapor pressures used in SSR to produce MCT can cause the ampoule to explode. Also, very long annealing times are required and the resulting crystals are small. The typical diameter of the ingot is limited to about $2.0 \mathrm{~cm}$ in order to control impurity segregation in the crystal.

\subsubsection{Traveling Heater Method}

In the THM method, a solvent zone is created between a solid seed and the feed stock material. In the case of $\mathrm{MCT}$, the crystal is grown by passing the solvent zone (e.g. Te rich) through a polycrystalline MCT rod having a composition that is to be replicated in grown crystal. The motion of the molten interface is produced by the slow movement of the heater along the charged crucible. Crystallization takes place at the advancing seed-solvent interface and dissolution of feed material occurs at the solvent-feed phase boundary. Through 
convection and diffusion, the solid material is dissolved at the high temperature interface and deposited at the low temperature interface of the zone.

Crystal growth occurs in the $500-700{ }^{\circ} \mathrm{C}$ range, lower than the temperature used for Solid State Recrystallization growth method. The lower growth temperature used in THM reduces the incidence of antisite defects, resulting in crystals with more reproducible composition and higher resistivity. Also, the lower temperatures reduce contamination from the crucible walls and decrease the evaporation of the constituent species. One successful implementation of THM resulted in crystals up to $5 \mathrm{~cm}$ in diameter [15]. The perfect quality of crystals grown by this method is achieved at the cost of a low growth rate [11].

\subsubsection{Liquid Phase Epitaxy (LPE)}

LPE growth method offers, in comparison with bulk growth techniques, lower growth temperatures, shorter growth times, multilayered device structures, and better compositional homogeneity over large substrate areas. The versatility of LPE as a production tool for high performance device quality MCT epitaxial layers, with different Cd mole fractions and excellent compositional uniformity, is discussed in [16-20]. Today, detector arrays prepared from LPE based materials exhibit best performance, and majority of military IR applications use this technology.

LPE is a solution growth technique that involves the controlled precipitation of a solute dissolved in a solvent onto a single crystal substrate. For LPE growth of MCT, bulk grown CdZnTe single crystal substrates are suitable, since they are thermodynamically compatible and nearly lattice matched. The solvent can either be $\mathrm{Hg}$ [21] or Te-rich [22].

Both Te-solution growth $\left(420-500{ }^{\circ} \mathrm{C}\right)$ and $\mathrm{Hg}$-solution growth $\left(360-500{ }^{\circ} \mathrm{C}\right)$ are used with equal success in a variety of configurations. The design of the Te-rich LPE system can be configured to allow the melt to contact the substrate by either sliding, tipping or dipping techniques. A sliding boat system uses a small melt volume and is adaptable for changing composition, thickness and doping. Tipping and dipping systems can be scaled up for large melts to provide thick, uniform layers. Both the tipping and dipping designs are being used for Te and Hg-rich solutions, while only the sliding technique is used for Te-rich solutions.

The major difference between the $\mathrm{Hg}$ and Te-rich solvents is that in the former case, the vapor pressure of $\mathrm{Hg}$ over the melt is much higher than in the latter case. The $\mathrm{Hg}$ partial pressure curves in Figure 4 indicate that at $500{ }^{\circ} \mathrm{C}$ and a $\mathrm{Cd}$ mole fraction of 0.1 , the $\mathrm{Hg}$ partial pressure over Te-saturated MCT is $0.1 \mathrm{~atm}$, while that of $\mathrm{Hg}$-saturated MCT is $7 \mathrm{~atm}$ [23]. Te-rich solutions saturated with $\mathrm{Hg}$ vapor allow for small volume melts that do not appreciably deplete during growth in the temperature range $420-500^{\circ} \mathrm{C}$ using the slider technique.

This is because the solubility of $\mathrm{Cd}$ in Te is high. On the other hand, the limited solubility of $\mathrm{Cd}$ in $\mathrm{Hg}$ requires the volume of $\mathrm{Hg}$-rich melts to be much larger than Te melts, in order to minimize melt depletion during growth in the $360-500{ }^{\circ} \mathrm{C}$ temperature range. Unfortunately, the larger melt volume in Hg-rich LPE precludes the use of the slider boat approach and makes an open tube growth impossible [24]. For these reasons, it would not be surprising if many more manufacturers are pursuing LPEgrowth from Te-rich melts rather than from $\mathrm{Hg}$-rich melts. 


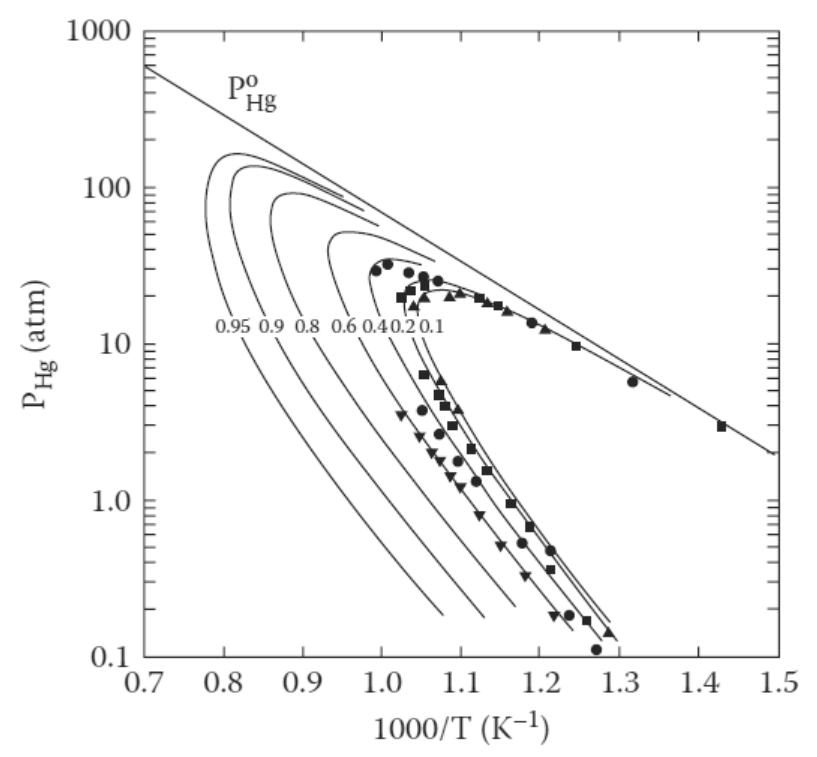

Figure 4. Partial pressure of $\mathrm{Hg}$ along the three-phase curves for various MCT solid solutions [23].

Despite the lower $\mathrm{Hg}$ vapor pressure in a Te-rich melt, the partial pressure of $\mathrm{Hg}$ must still be controlled in the growth system in order to obtain compositional uniformity, reproducibility, and stability. One way of controlling the $\mathrm{Hg}$ partial pressure under Te-rich conditions is to carry out the entire LPE growth process in a sealed ampoule. The disadvantages of this approach are low production levels because of the necessity of sealing the ampoule for each growth run and difficulty for in-situ preparation of multilayer growth that is necessary for advanced IR device structures.

In an open tube LPE-MCT, Te-rich system, the Hg partial pressure can be controlled by [25]: a) implementing an external $\mathrm{Hg}$ source to replenish the depleted $\mathrm{Hg}$ from the growth chamber, b) using chunks of HgTe near the melt as a solid source for $\mathrm{Hg}$ vapor, or c) using a high inert gas overpressure to minimize $\mathrm{Hg}$ loss.

As discussed above, the growth of MCT from Hg-rich melts is not as popular as growth from Te-rich solutions because of the low solubility of $\mathrm{Cd}$ and $\mathrm{Te}$ in $\mathrm{Hg}$ below $600{ }^{\circ} \mathrm{C}$, and the high vapor pressure of $\mathrm{Hg}$. On the other hand, LPE growth from a Hg-rich melt offers the following advantages: excellent surface morphology; high purity source material; good control over N- and P-type doping levels; very good compositional and thickness uniformity over large surface areas; and no need for post-growth anneals.

LPE layers grown from Te-rich melts are P-type due to the $\mathrm{Hg}$ vacancies induced during the growth process. These unintentionally doped layers can be converted to N-type by appropriate annealing schedules in $\mathrm{Hg}$ vapor. Layers grown from $\mathrm{Hg}$-rich melts are usually $\mathrm{N}$ type. LPE layers grown from Hg-rich solutions are intentionally doped with group VB 
elements with high solubilities [20], but layers grown from Te-rich solutions are not [26]. Group VB dopants have low solubility and are not fully active electrically. Group IIIB elements, indium in particular, are easily incorporated from both solutions. Indium doping from Te-rich melts, however, has the advantage that the segregation coefficient is near unity.

From a device perspective, its performance is dependent on the electrical, optical and mechanical characteristics of the epitaxial layers. For IR FPA, the dislocation count controls the number of defective pixels. Typical etch pit (chemical etched decoration of dislocations) densities for LPE layers grown from a Te-rich melt onto a CdZnTe substrate are in the $3-7 \mathrm{E} 4 / \mathrm{cm}^{2}$ range [27]. These defects are associated with threading dislocations that are normal to the epilayer surface. Compositional uniformity of $x=0.223+/-0.001$ has been demonstrated over areas ranging from $43-54 \mathrm{~cm}^{2}$. For a series of 200 growth runs, the run-to-run reproducibility in composition of $x=0.226+/-0.0033$ has been demonstrated [28].

\subsubsection{Metal-Organic Chemical Vapor Deposition (MOCVD)}

In general, MOCVD growth of MCT depends on transporting the elements $\mathrm{Cd}$ and $\mathrm{Te}$ (and dopants In, I and As) at room temperature as volatile organometallics. These species react with $\mathrm{Hg}$ vapor in the hot gas stream above the substrate or catalytically on the substrate surface at about $400{ }^{\circ} \mathrm{C}$. The pyrolytic nature of the reaction requires that only the substrate be heated to ensure efficient deposition. In practice, the growth of MCT by MOCVD using dimethyl cadmium (DMCd) and diethyl telluride (DETe) is accomplished by two processes: a) CdTe synthesis from DMCd and DETe, and b) formation of $\mathrm{HgTe}$ from DETe and $\mathrm{Hg}$ at the heated substrate.

The challenge with this growth technique is to control the composition of the epitaxial layer and achieve uniformity over large surface areas. MOCVD-MCT composition is influenced by substrate temperature, $\mathrm{DMCd}$ and $\mathrm{Hg}$ partial pressures. Compositional control and layer uniformity are addressed using the inter-diffused multilayer process (IMP) technique in which very thin layers $(0.1-0.2 \mu \mathrm{m})$ of $\mathrm{HgTe}$ and CdTe are deposited sequentially. These layers, with high diffusion coefficients, inter-diffuse during growth at about $400{ }^{\circ} \mathrm{C}$ to form a homogeneous ternary epilayer with a composition that is controlled by the thickness ratio of HgTe:CdTe layers [29].

The preferred precursor for Te is di-isopropyl telluride (DiPTe) that allows for a reduction in the MCT growth temperature from $400{ }^{\circ} \mathrm{C}$ to $350{ }^{\circ} \mathrm{C}$. DiPTe in conjunction with DMCd can allow the deposition CdTe to occur at lower temperatures $\left(300^{\circ} \mathrm{C}\right)$. Doping for MOCVDMCT layers is straightforward using Group III metals for P-type doping and Group VII halogens for N-type doping. The main morphological problem for MOCVD are macro defects called hillocks, which are caused by preferred (111) growth, nucleated from a particle or polishing defect. Hillocks can cause clusters of defects in focal plane arrays. Orientations 3-4 off (100) are used primarily to reduce both the size and density of hillocks.

The MOCVD technique is used to manufacture high-quality, large-area infrared focal plane arrays for many applications [30]. Consequently, there is a renewed interest in using MOCVD because of its ability to: achieve low surface defect densities, deposit MCT films on large area- 
low cost substrates such as GaAs and control N- and P-type doping levels. In comparison to an MBE system, the overall maintenance and operational costs of an MOCVD system is lower.

\subsubsection{Molecular Beam Epitaxy (MBE)}

Thin film deposition by MBE enables the growth of large area epilayers with sophisticated multilayer structures having abrupt and complex compositions and doping profiles. Growth of MBE-MCT is carried out under an ultra-high vacuum environment with Knudsen-type effusion source cells charged with $\mathrm{Hg}$, Te2, and CdTe [31-32]. MBE-MCT deposition temperature plays a critical role in the introduction of extended defects. Typically, growth is carried out at $180^{\circ} \mathrm{C}-190^{\circ} \mathrm{C}$ on (211) CdZnTe substrates.

The low growth temperature and the ability to rapidly shutter the sources are key features that allow MBE to produce sharp interfaces for multilayered IR devices that operate in two or three different spectral bands. The ultra-high vacuum growth chamber allows for in-situ analytical tools to monitor and control the MCT growth process and evaluate the properties of the grown layers [33-34].

At the lower temperature range, a Hg-rich condition prevails at the substrate because the sticking coefficient of $\mathrm{Hg}$ increases as the temperature is reduced. The condition with excess $\mathrm{Hg}$ results in the formation micro-twins that are detrimental to the performance of the MCT IR focal plane array. Typical etch pit densities (EPD) of material grown under such Hg-rich conditions are high $\left(10^{6}-10^{7} \mathrm{~cm}^{-2}\right)$. If the growth temperature is raised to about $190{ }^{\circ} \mathrm{C}$, then a deficiency of $\mathrm{Hg}$ leads to the formation of voids in the MCT layer.

$\mathrm{Hg}$ is incorporated in the film only by reacting with free Te, thus the MCT composition is contingent on the Te to CdTe flux ratio. The structural perfection of the film depends strongly on the $\mathrm{Hg}$ to $\mathrm{Te}$ flux ratio and growth is usually restricted to a tight temperature range. By optimizing the $\mathrm{Hg}$ to Te flux ratio, the concentration of voids is about $100 \mathrm{~cm}^{-2}$ which may be attributable to dust particles or substrate related imperfections. The EPD values for epilayers grown under these conditions are in the low $10^{5} \mathrm{~cm}^{-2}$ ranges.

Indium is the most widely used N-type extrinsic dopant in MCT epitaxial layers and is well activated. At low Indium doping levels, $\mathrm{Hg}$ vacancies can compensate some of the N-type impurities and affect dopant control. P-type dopants, such as Arsenic, are less conveniently incorporated into the epilayer. Significant efforts are being expended to improve the incorporation of $\mathrm{As}$ and $\mathrm{Sb}$ during the MBE process and to reduce the temperature required for activation. The metal saturation conditions cannot be reached at the temperatures required for high-quality MBE growth. The necessity to activate acceptor dopants at high temperatures diminishes the gains of low-temperature deposition. Nearly $100 \%$ activation has been achieved for a $2 \times 10 \mathrm{E} 18 \mathrm{~cm}^{-3}$ As concentration, with as low as $300{ }^{\circ} \mathrm{C}$ activation anneal, followed by a $250{ }^{\circ} \mathrm{C}$ stoichiometric anneal [35].

Because of its various advantages, MBE-MCT technology is becoming more attractive than the other epitaxial technologies and is required for the fabrication of IR detectors with advanced architectures. The MBE-MCT technology has developed to the point where MBE lay- 
ers grown on bulk CZT substrates exhibit characteristics comparable to those prepared by LPE. MBE technology is now routinely used for multicolor detector arrays.

The main challenge of MBE-MCT technology is to grow very high quality layers on lowcost, large- area substrates. The issues that complicate MBE growth on alternative large-area substrates are: lattice mismatch, nucleation phenomena, thermal mismatch, and contamination [36-37]. Sapphire, Si, and GaAs are some of the low-cost, large-area materials that have been successfully employed as substrates for MCT epitaxial growth [38-41]. However, appropriate buffer layers of CdTe or CZT are required on the alternative substrates before MCT films are deposited.

The best MBE-MCT layers grown on buffer/Si substrates achieved thus far exhibit defect densities of $2-5 \times 10^{6} \mathrm{~cm}^{-2}$. Novel thermal cycle annealing schedules have been used to further reduce the defect density.More effort is necessary to reduce this defect density by at least an order of magnitude to make MBE based materials for many military applications. The ability to grow MCT on large diameter Si wafers will enable low cost, large format infrared focal plane arrays.

\section{SWIR Detector Technologies}

The SWIR band (0.9-2.5 um) bridges the spectral gap between the visible and thermal bands in the electromagnetic spectrum. In this spectral band, the primary phenomenology of interest is the reflectance signature of the target, manifested as either its variations in brightness or spectral reflectance, or both.

Infrared imaging in the SWIR band offers several advantages: can detect reflected light, offering more intuitive, visible-like images; better suited for imaging in adverse environments and weather conditions, including fog, dust, and smoke; can also see in low light conditions, and use eye safe $1550 \mathrm{~nm}$ illumination that is totally undetectable by regular night vision equipment; and can generate digital video outputs and thus offering more advantages than traditional image intensifier night vision equipment. Under low light conditions, the sensitivity of the focal plane array is ultimately determined by the $\mathrm{R}_{0} \mathrm{~A}$ product of the photodiode.

\section{1. $\operatorname{In}_{\mathrm{x}} \mathrm{Ga}_{1-\mathrm{x}}$ As Detector Array Development}

For SWIR imaging, InGaAs is one of the widely used detector materials due to its low dark current. The detector material can be prepared using any of the following techniques: Molecular beam epitaxy (MBE), metal-organic chemical vapor deposition (MOCVD), liquid phase epitaxy (LPE), hydride-transport vapor phase epitaxy (VPE), and atomic layer epitaxy (ALE). InGaAs layers are typically grown on lattice matched InP substrates using an alloy composition of $\mathrm{x}=0.53$.

The spectral response typically covers $0.9-1.7 \mu \mathrm{m}$ at room temperature. By increasing the composition to $\mathrm{x}=0.82, \mathrm{InGaAs}$ is able to extend its cutoff to $2.6 \mu \mathrm{m}$. However, the crystal 
defects due to epitaxy and the decreased shunt resistance, due to a smaller band gap, degrade performance at the longer cutoff wavelengths.

The band gap [42] of the strained $\operatorname{In}_{x} \mathrm{Ga}_{1-x}$ As:InP structure can be tailored by varying the alloy composition during crystal growth according to the equation:

$$
E_{g}(e V)=\left(E_{g_{\text {GaAs }}}-\frac{\alpha_{\text {GaAs }} T^{2}}{T+\beta_{\text {GaAs }}}+\left(E_{g_{\text {lnAs }}}-\frac{\alpha_{\text {InAs }} T^{2}}{T+\beta_{\text {InAs }}}-E_{g_{\text {GaAs }}}+\frac{\alpha_{\text {GaAs }} T^{2}}{T+\beta_{\text {GaAs }}}\right) x-0.475 x(1-x)\right.
$$

Where $\mathrm{E}_{\mathrm{g}}$ is the band gap in $(\mathrm{eV}), \alpha$ and $\beta$ are fitting parameters, and $\mathrm{x}$ is the In:As ratio. The cut-off wavelength can be calculated from the expression $\lambda_{\mathrm{co}}=\mathrm{hc} / \mathrm{E}_{\mathrm{gap}}$.

The response can be extended to include the visible wavelength range by removing the InP substrate. There has been an intensive effort to develop InGaAs arrays for Low Light Level (LLL) SWIR imaging [42-47]. An example is in astrophysical space based observatories that are very demanding on the detectors due to the very low IR flux levels. Such low flux levels represent the detection of few photons over long integration times and, therefore, require extremely low dark current photodiodes hybridized to a high performance ROIC stage. For such LLL applications there are challenges ahead to further lower noise, reduce pixel size, fabricate larger arrays, achieve higher operating temperatures, and reduce production cost.

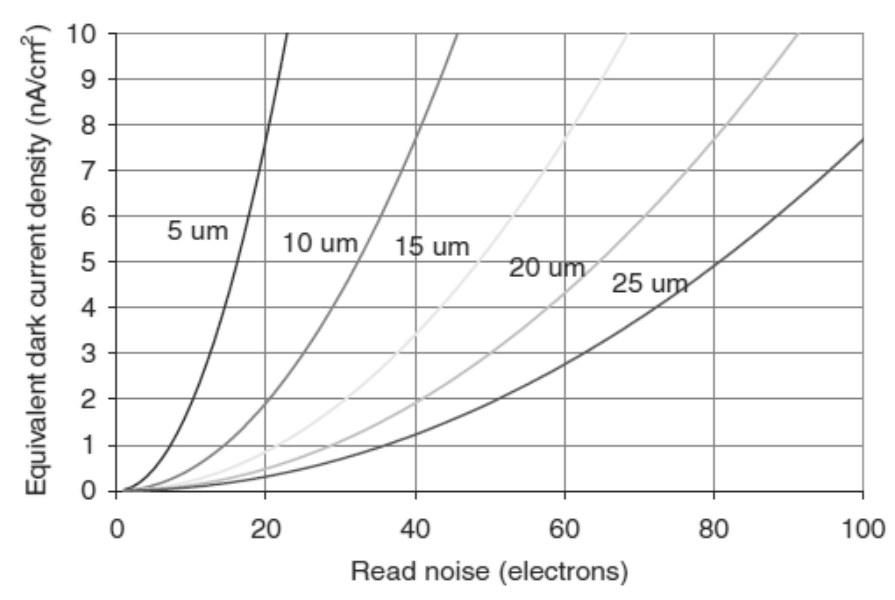

Figure 5. Dark current density versus read noise for different pixel pitches [46].

The spectral response of InGaAs diodes at room temperature is in the $0.9-1.67 \mu \mathrm{m}$ wavelength range which matches the ambient night glow spectrum. Imaging under such low light conditions requires that the noise of the detector be extremely low. A significant portion of the noise is contributed by the dark current of the InGaAs detector and the readout 
noise. Dark current consists of unwanted thermally generated carriers that can cause the detector to produce a random varying output signal.

It is associated with interfacial, diffusional, G-R, and tunneling currents. The temperature dependence of the dark current is primarily due to the intrinsic carrier concentration which depends exponentially on the temperature. The dark current of the detector can be reduced through appropriate fabrication processes and device design. The impact of dark current noise as a function of read noise is shown in Figure 5, where the curves for different pixel pitch map the dark current noise into an equivalent read noise.

For a given read noise, the required dark current density increases as the pixel pitch is decreased. The challenge is to maintain a low dark current density as the pixel pitch is reduced. Simultaneously, the challenge for the read out circuit is to reduce the read noise. If the limitation is due to the detector and its noise level overwhelms the source signal, the solution may be to use an external illuminator or cool the detector. The choice of either solution will depend on a tradeoff between size, weight, and power requirements (SWaP).

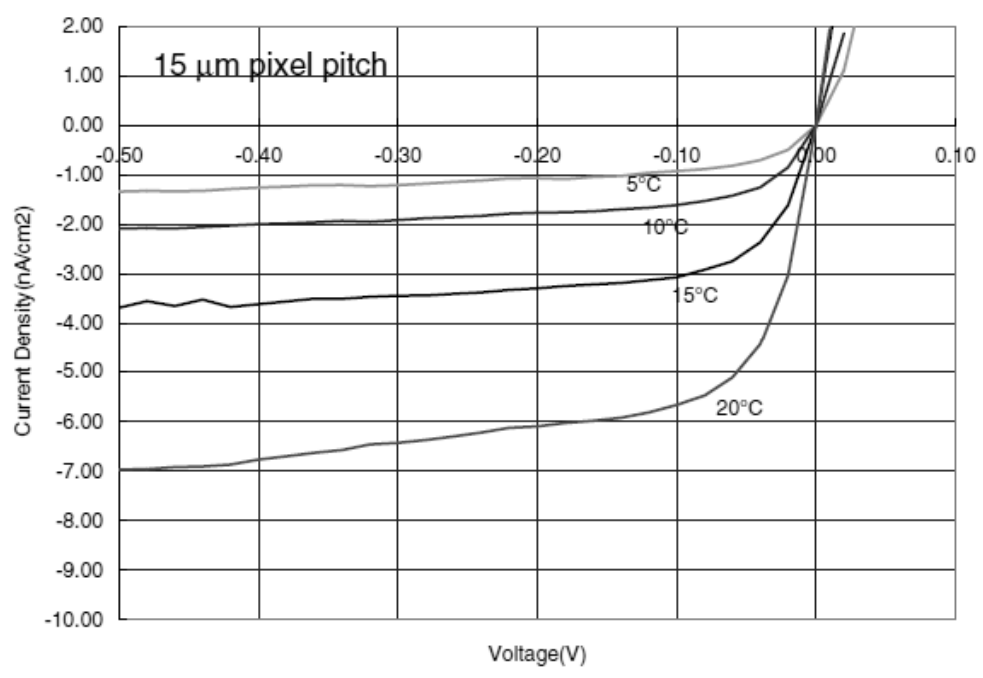

Figure 6. Dark current density at different temperatures using test structures on the wafer. Test arrays have 225 pixels (15 $\mu \mathrm{m}$ pitch) and the guard ring is not biased [46].

Applications involving situational awareness require FPAs to have more pixels (large format/high resolution) for increased surveillance coverage. For soldier portable and some airborne platforms, it is desirable to reduce the size of the pixel in order to satisfy the constraints of low SWaP and cost, without sacrificing performance. However,as the pixel pitch is scaled to smaller geometries, there is a tendency for the dark current density to increase. The reduced pixel diameter can cause the sidewall related dark current to become more pronounced and overwhelm the area related contribution, resulting in 
an effective increase in the dark current density. The sidewall contribution can be avoided with appropriate surface passivation of the exposed PN junction.

As mentioned above, the dark current of the detector can be reduced through appropriate fabrication processes and device designs. By focusing on the growth conditions for the InGaAs absorption layer, heterointerfaces and the passivation layer, researchers have been able to demonstrate dark current densities below $1.5 \mathrm{nA} / \mathrm{cm}^{2}$ at $7{ }^{\circ} \mathrm{C}$ and a bias of $100 \mathrm{mV}$ for $15 \mu \mathrm{m}$ pitch InGaAs arrays as shown Figure 6.
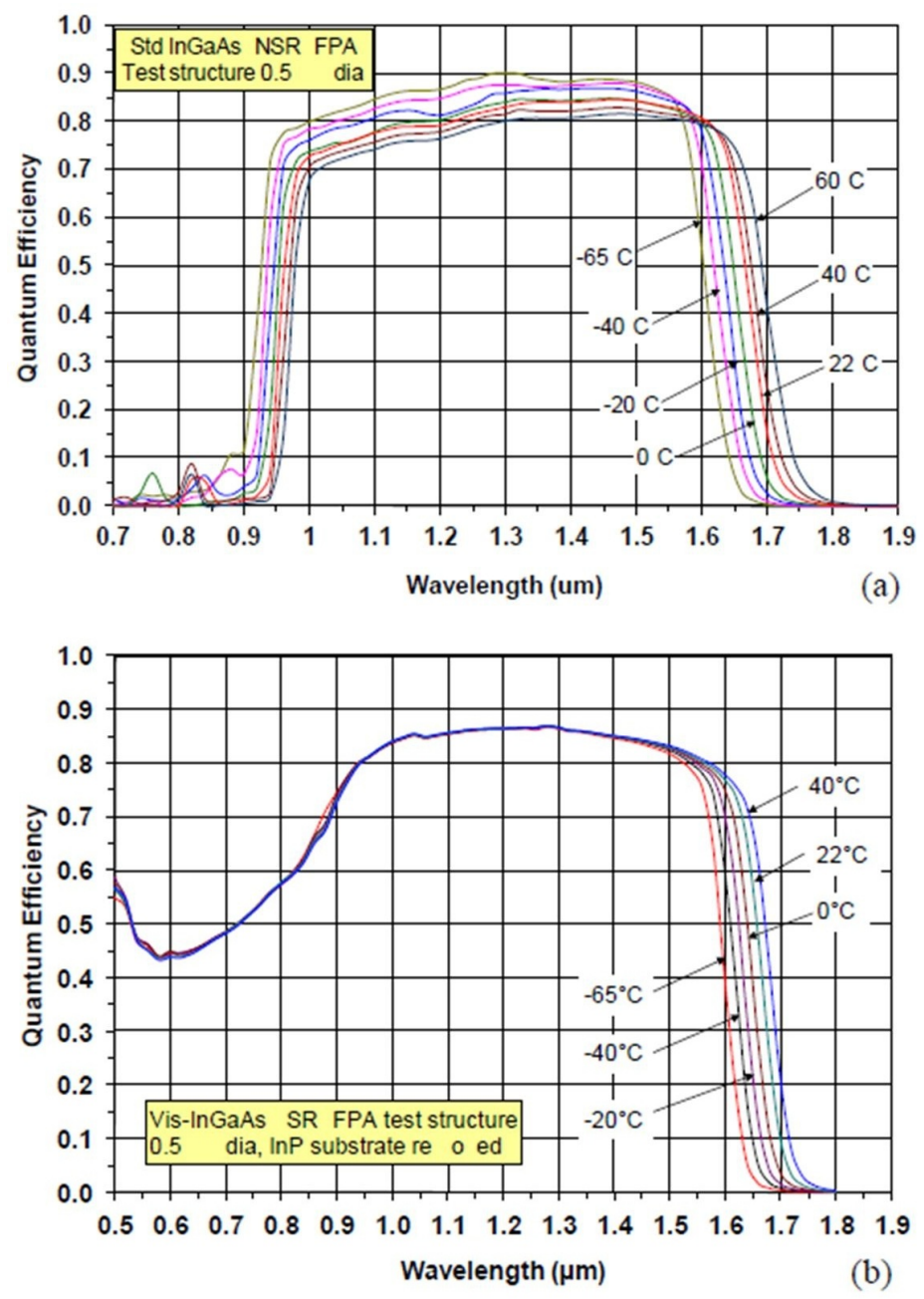

Figure 7. Spectral QE vs. wavelength at different temperatures measured for backside illuminated InGaAs photodiodes test array demonstrating Visible-Near IR response with a) InP substrate not removed and b) InP removed [47]. 
To achieve the constraints of low SWaP and cost, manufacturers are now developing InGaAs detectors on 4" diameter wafers. For example, 16 - 1280x1024 InGaAs arrays with 15 $\mu \mathrm{m}$ pixels have been demonstrated on 4" InP wafers. To extend the spectral response of these detector arrays down to the UV band, the InP substrates are removed [47]. The test results for a backside illuminated $0.5 \mathrm{~mm}$ InGaAs detector is shown in Figure 7 [47]. The Quantum Efficiency (QE) achieved across the 1.2-1.6 $\mu \mathrm{m}$ band is about $80 \%$ over a temperature range of $-65{ }^{\circ} \mathrm{C}$ to $40{ }^{\circ} \mathrm{C}$.As a result of removing the $\mathrm{InP}$ substrate (see Figure 7 (b)), the $\mathrm{QE}$ is about $40 \%$ over the entire visible band.

Continued effort is underway to demonstrate large format $(>2 \mathrm{~K} \times 2 \mathrm{~K})$ and small pixel $(<10$ $\mu \mathrm{m})$ InGaAs FPAs fora variety of room temperature, low light level (LLL) imaging applications, such as night vision. These applications demand extremely low detector dark current and reduced ROIC noise to maintain performance, since the photon collection area is reduced [47].

\subsection{SWIR $\mathrm{Hg}_{1-\mathrm{x}} \mathrm{Cd}_{\mathrm{x}} \mathrm{Te}(\mathrm{MCT})$ Detector Arrays}

Another approach to accomplish SWIR imaging under low light level (LLL) conditions is to use MCT detectors grown by either MBE or LPE techniques. For $\mathrm{Hg}_{1-\mathrm{x}} \mathrm{Cd}_{\mathrm{x}} \mathrm{Te}$, the alloy composition can be fixed to provide an energy band gap equal to the longest wavelength to be measuredin the SWIR band. The larger energy band gap enables higher operating temperatures; MCT arrays operating at near $150 \mathrm{~K}$ have achieved BLIP limits at background levels as low as $10^{11}$ photons $/ \mathrm{sec} / \mathrm{cm}^{2}$ [48]. There are continued efforts to increase the operating temperature of SWIR MCT detectors [49].

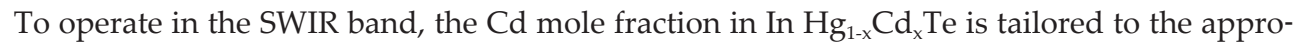
priate energy band gap [50] according to the expression:

$$
E g(e V)=-0.302+1.930 x+5.35 \times 10^{-4} T(1-2 x)-0.810 x^{2}+0.832 x^{3}
$$

The absorption coefficient of MCT is very large and in order to have high responsivity, the rule of thumb is that the thickness of the MCT absorber layer should be at least equal to the cutoff wavelength. For the MCT material system, the choice of P-on-N polarity is generally driven by dark current considerations. The presence of $\mathrm{Hg}$ vacancies in the $\mathrm{P}$ base layer of an N-on-P diode degrades the minority carrier lifetime, resulting in larger dark currents. Nevertheless, to meet very low dark current requirements, diodes can be cooled down to very low temperatures at the expense of SWaP.

Figure 8 presents results for ion implanted P-on-N (MBE with $\mathrm{N}_{\mathrm{D}}=1 \mathrm{E}^{16} \mathrm{~cm}^{-3}, 4-\mu \mathrm{m}$ thick) and N-on-P (LPE with $\mathrm{N}_{\mathrm{A}}=3-5 \mathrm{E}^{16} \mathrm{~cm}^{-3}, 7 \mu \mathrm{m}$ thick) diodes fabricated on lattice matched CZT in order to ensure a low dislocation density $\left(\operatorname{mid} 10^{4} / \mathrm{cm}^{2}\right)$. Diodes are based on planar technologies, with CdTe and ZnSe passivation layers [51]. Very low, state of the art dark currents are observed over a wide temperature range as shown in Figure 8. 

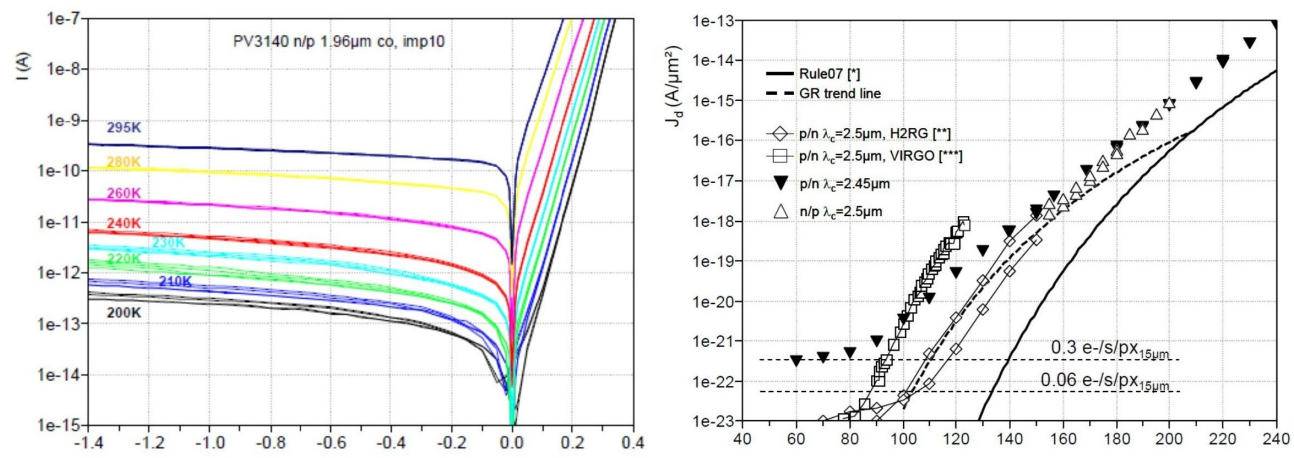

Figure 8. a) I-V curves measured on isolated $10 \mu \mathrm{m}$ N-on-P implanted diodes; Cutoff=2.0 um. b) Measured dark currents and comparison with literature data at $2.5 \mu \mathrm{m}$ cut-off [51].

\section{3. $\mathrm{Si}_{1-\mathrm{x}} \mathrm{Ge}_{\mathrm{x}}(\mathrm{SiGe})$ Detector Arrays}

Like the other two alloy semiconductors mentioned above, SiGe is another example of material that can be used for the fabrication of SWIR detectors. The key attractive feature of SiGe IR detectors is that they can be fabricated on large diameter Si substrates with size as large as 12-inch diameter using standard integrated circuit processing techniques. Furthermore, the SiGe detectors can be directly integrated onto low noise Si ROICs to yield low SWaP, low cost and highly uniform IR FPAs. The primary motivation for SiGe SWIR FPA development is the CMOS-like fabrication allowing for very low cost technology.

Some of the earlier attempts in developing SiGe IR detectors focused on their LWIR applicationsby using internal photoemmision [52-53]. Renewed efforts are now developing these detectors for application in the NIR-SWIR band [54]. For the SiGe material to respond to the SWIR band, its cutoff wavelength is tuned by adjusting the SiGe alloy composition. Si and Ge have the same crystallographic structure and both materials can be alloyed with various Ge concentration. The lattice constant of $\mathrm{Ge}$ is $4.18 \%$ larger than that of $\mathrm{Si}$, and for a $\mathrm{Si}_{1-\mathrm{x}} \mathrm{Ge}_{\mathrm{x}}$ alloy ("a" for alloy) the lattice constant does not exactly follow Vegard's law. The relative change of the lattice constant is given by [55]:

$$
\mathrm{aSi}_{1-\mathrm{x}} \mathrm{Ge}_{\mathrm{x}}=0.5431+0.01992 \mathrm{x}+0.0002733 \mathrm{x}^{2}(\mathrm{~nm})
$$

For a $\mathrm{Si}_{1-\mathrm{x}} \mathrm{Ge}_{\mathrm{x}}$ layer with $\mathrm{x}>0$ on a $\mathrm{Si}$ substrate means that the layer is under compressive stress. A perfect epitaxial growth of such a strained heteroepitaxial layer can be achieved as long as its thickness does not exceed a critical thickness for stability. Beyond the critical thickness, the strain is relaxed through the formation of misfit dislocations, which can cause an increase in the dark current. Several approaches have been proposed to reduce the dark current in SiGe detector arrays by several orders of magnitude.These include fabrication methodologies, de- 
vice size and novel device architectures, such as Superlattice, Quantum dot and Buried junction designs [54]. Furthermore, some of these approaches have the potential of extending the wavelength of operation beyond 1.8-2.0 microns. The challenge is to take advantage of these innovative device designs and reduce the dark currents to $1-10 \mathrm{nA} \mathrm{cm}^{-2}$.

A proposed diagnostic device structure to evaluate the impact of various fabrication methodologies to reduce leakage currents and produce higher detector performance in $\mathrm{SiGe} / \mathrm{Si}$ is shown in Figure 9. The structure can help to assess the following: ability to grow high quality/low defect density Ge on $\mathrm{Si}$; layer thickness necessary for minimal topological and defect density requirements; isolation of defect states at the Ge/oxide interface from the signal carrying layers; and optimum doping and thickness of the P-type Ge layer under the oxide to isolate interface states and lateral leakage current that could result between the highly doped $\mathrm{N}^{+}$Ge region used for contacts and the lighter doped P-type Ge region.

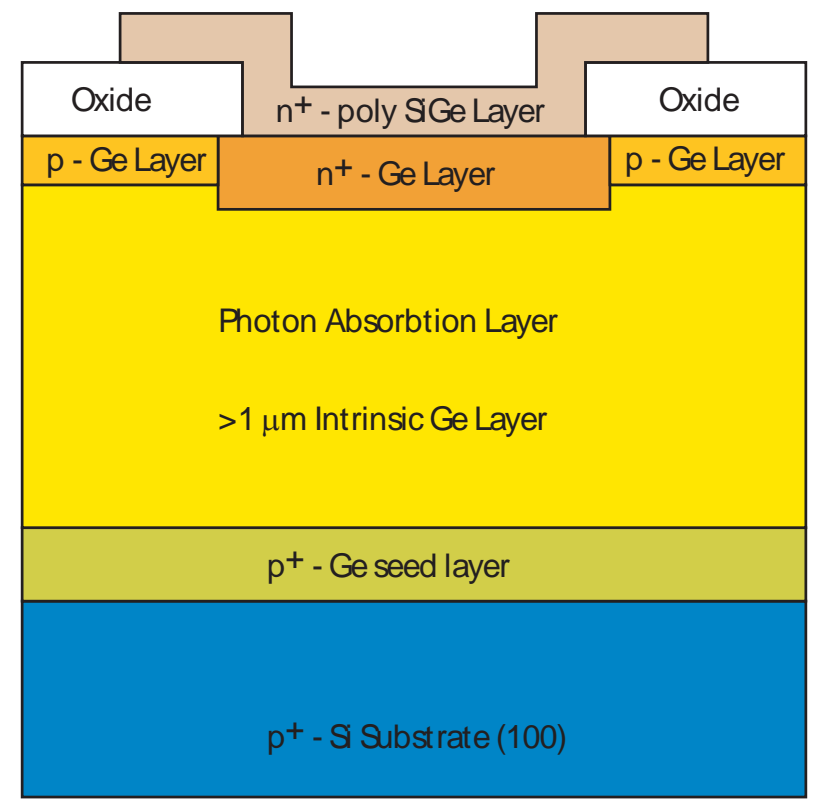

Figure 9. SiGe/Si diagnostic structure to evaluate impact of various fabrication methodologies.

Dark currents in SiGe detectors can be reduced by reducing the pixel size, since dark currents track with thevolume of the pixel. Reductions in size are advantageous for resolution; however, for low light level conditions, such as nightglow, a large pixel size or at least a large collection area is required. The I-V characteristics of photodiodes with different areas fabricated on $2 \mu \mathrm{m}$ thick intrinsic epitaxial Ge layers are shown in Figure 10(a). The curves indicate that the dark current is lowered as the device area is reduced. The responsivity as a function of wavelength for a $100 \mu \mathrm{m} \times 100 \mu \mathrm{m}$ diode without an anti-reflection coating is given in Figure 10(b). The reverse leakage currentat a reverse bias of 1 vol tis $32 \mathrm{~mA} / \mathrm{cm}^{2}$. 

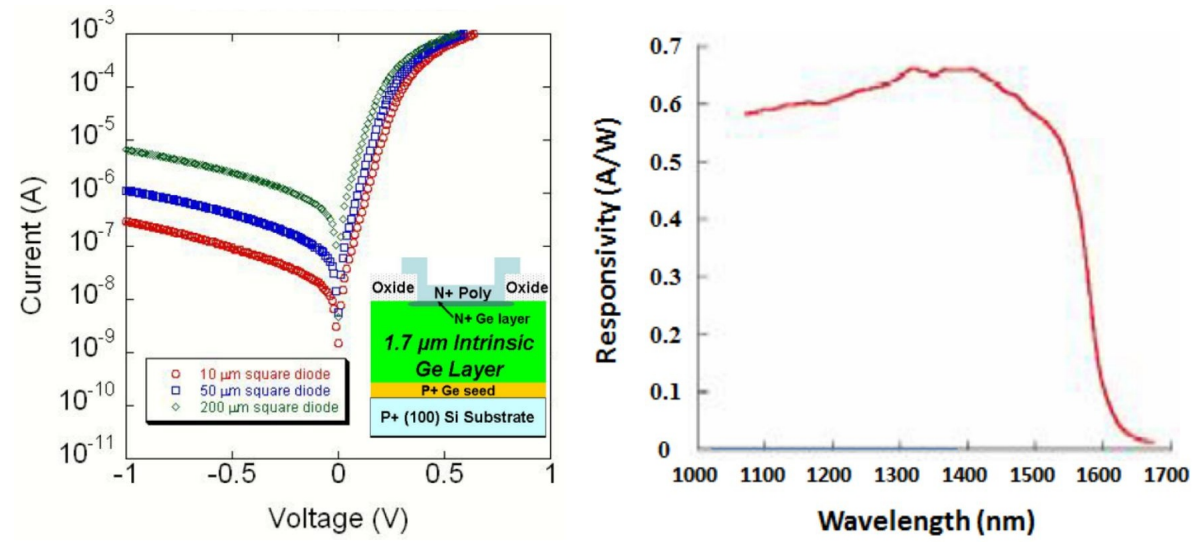

Figure 10. a) Measured room temperature I-V characteristics for large area diodes with 20, 50 and 200 micron unit cell. The inset shows the schematic device cross section. b) The spectral response data for SiGe detector [56].

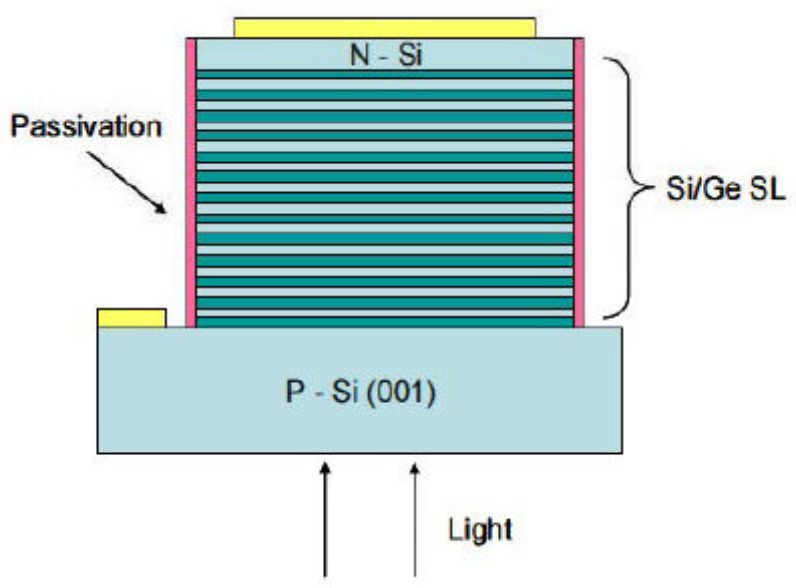

Figure 11. Schematic of detector array structure consisting of a SiGe /Si strained layer Superlattice grown on (001) silicon [54].

Figure 11 shows the Strained-Layer Superlattice (SLS) structure being evaluated for longer detector array response to 2 microns. It consists of SiGe quantum wells and Si barrier layers, grown on p-type (001) Si substrates. Super lattices having differing Si barrier and Ge well thicknesses to control the strain are grown to optimize wavelengthresponse and dark current. The SiGe well thicknesses are kept below the critical layer thickness fordislocation formation. To complete the structure, the undopedsuperlattice is capped with a thin $\mathrm{n}+\mathrm{Si}$ caplayer to form the p-n junction. After growth the devices are patterned with a top contact, 
mesas are etched to provide isolation and the substrate contact is formed. The etched mesa can also be passivated to minimize surface recombination as indicated in Figure 11. The device shown in Figure 11 uses substrate sideillumination, as is needed for use in FPA arrays, and short wavelength response can be improved by thinning the Si substrate.

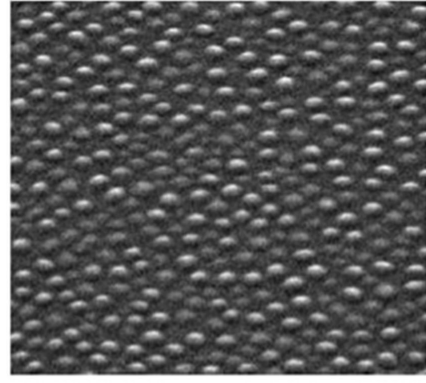

(a)

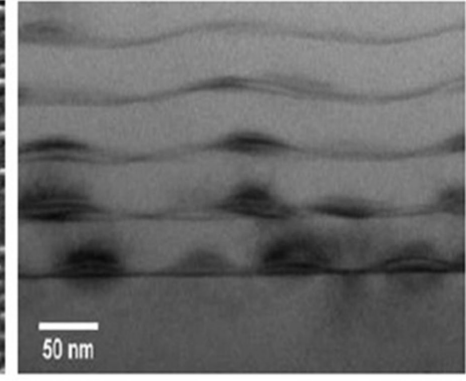

(b)

Figure 12. (a) SEM image ( $45^{\circ}$ tilt) of a Ge QD layer deposited on Si. The QDs are $\sim 60 \mathrm{~nm}$ in diameter with a density of $10^{20} / \mathrm{cm}^{2}$. (b) Cross-sectional TEM image of Ge/Si QDSL. Ge QDs appear with dark contrast compared to Si barriers [54].

The strained-layer superlattice and quantum dot superlattice (QDSL) in the SiGe material system have the potential of developing Vis-NIR detector arrays with longer cutoff wavelength and potentially lower dark current. The advantage of quantum dots is the potential to exploit the optical properties of Ge while avoiding dislocation formation. Ge QDs grown on $\mathrm{Si}$ in Stranski-Krastanov mode can be deposited well beyond the critical thickness without dislocation nucleation [56].

Figure 12 (a) shows the SEM image of an array of Ge nanodots grown by MOCVD. These dots are typically $50-75 \mathrm{~nm}$ in diameter with area coverage of $\sim 20 \%$. To increase optical absorption and sensitivity, MOCVD-based growth techniques are being developed for the deposition of $\mathrm{Ge} / \mathrm{Si}$ quantum dot superlattices (QDSLs), where Ge QDs are alternated with thin (10-30 nm) Si barrier layers. A cross-sectional TEM image of QDSLs is shown in Figure 12(b).

\section{MWIR/LWIR Detector Array Technologies and Applications (InSb, HgCdTe, HOT, SLS and Bolometers)}

Most objects in earth's environment emit radiation in the MWIR/LWIR wavelength range, commonly referred to as the thermal band. For example, the human body, by virtue of being at a temperature of $\sim 300 \mathrm{~K}$, emits radiation that peaks around 10 microns. Also, most chemical species have spectral signatures in this infrared regime due to fundamental absorption processes associated with vibrational states of the molecules. Thus, in many applications that require the observation and identification of chemical species using point detection or standoff detection, such as pollution monitoring, gas leak detection, gas sensing and spec- 
troscopy, one needs to work in the MWIR/LWIR bands. Another distinct advantage is that the atmosphere has clear transmission windows in the MWIR and LWIR bands, making it very attractive for terrestrial applications.

\subsection{InSb Detector Array}

InSb detector arrays have found many applications in MWIR due to their spatial uniformity, low dark current and image quality. This technology has evolved over the years in response to the stringent requirements for applications in missile seekers and missile warning systems (MWS) [57-58]. For these applications, the IR imagers need to exhibit high dynamic range, fast frame rates, high resolution, very wide fields of view (FOV), and high sensitivity. The wide FOV optical design must consider the large incident angle of incoming photons, which if not included, can cause the appearance of ghost images and imaging of strong illumination sources outside the FOV. High spatial resolutions are achieved by using large arrays ( 640x512 and 1280x1024) with small pixels (unit cell size of $15 \mu \mathrm{m}$ ). The bandgap of this binary alloy is relatively constant and cannot be varied much as is the case with $\mathrm{HgCdTe}$ or SLS devices. Hence, to use InSb for high sensitivity multicolor applications requires the incorporation of filters to select bands of interest.

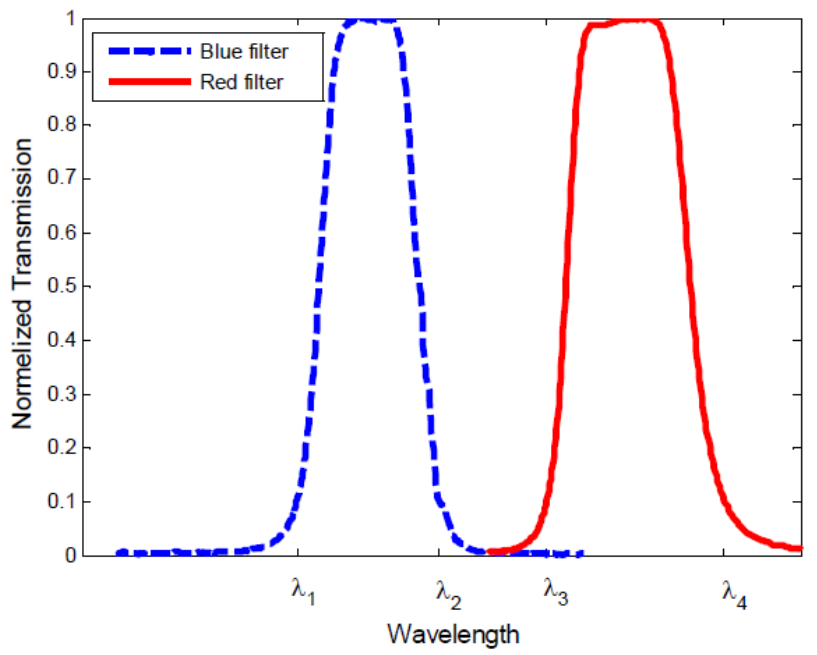

Figure 13. Normalized transmission of the cold filter in MWIR bands that are commonly referred to Blue (MWIR -1) and Red (MWIR-2) [58].

Many of the missile applications in the MWIR band require the use of two color InSb detectors, in order to discriminate the missile signature from the clutter background and reduce the false alarm rate. One band detects the target while the other band subtracts the background for noise suppression. The actual wavelength bands in 3-5 micron range vary from application to application [58]. 
Figure 13 presents the transmission of the integrated InSb sensor with cold filters for the two MWIR bands that are commonly referred to as the blue band (shorter wavelength) and the red band with the longer spectral response. The optical coatings on the detector arrays and the optics are designed for maximum transmission and to minimize the spectral crosstalk between the bands, typically less than $0.1 \%$, as can be seen in Figure 13 .
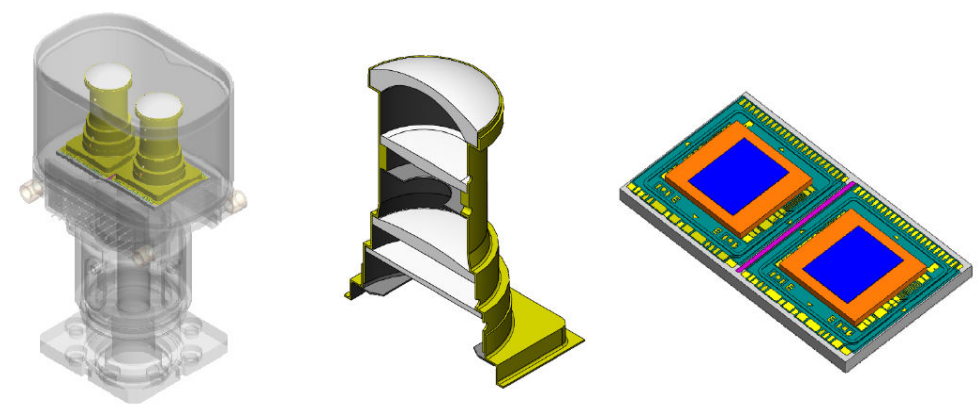

Figure 14. Dual-color Integrated Detector Cooler Assembly (IDCA) with two InSb FPAs and two cold radiation shields with integrated optics [58].

Figure 14 presents the schematics of various key building blocks for a Dual-color Integrated Detector Cooler Assembly (IDCA) with two InSb FPA's connected to their circuit card assemblies. Each of the InSb focal plane arrays has been optimized for the blue and red bands of interest in the broad MWIR region. As a dual band system, both FPAs can operate simultaneously at high frame rates.

\subsection{Multicolor HgCdTe (MCT) Detectors Arrays for MWIR/LWIR Applications}

MCT is the material of choice for a variety of high performance IRFPA systems for a variety of defense and commercial applications. Many of these applications use state-of-the-art $\mathrm{HgCdTe}$ growth using a bulk Cadmium ZincTelluride (CdZnTe) substrate. However, as the push for larger array sizes continues, it is recognized that an alternative substrate technology for large area needs to be developed for HgCdTe IRFPAs.

A significant effort has been under way in developing CdTe/Si or GaAs as a desired substrate. This substrate technology has been successful for short-wavelength (SWIR) and mid wavelength (MWIR) focal plane arrays; current $\mathrm{HgCdTe} / \mathrm{Si}$ material quality is being further developed for long-wavelength (LWIR) arrays, due to the high density of dislocations present in the material [59]. To remedy the high dislocation counts, researchers are focusing on both composite substrate development and improvement, and on $\mathrm{HgCdTe} / \mathrm{Si}$ post-growth processes [60]. The impact of ex-situ annealing on the quality of the epitaxial surface is shown in the Figure 15 for $\mathrm{HgCdTe} / \mathrm{Si}$ substrates. Several groups have demonstrated $\mathrm{HgCdTe} / \mathrm{Simaterial}$ with dislocation density measuring $1 \times 10^{6} \mathrm{~cm}^{-2}$. This is a five times reduction in the baseline material dislocation density that is currently used in the fabrication of devices. 

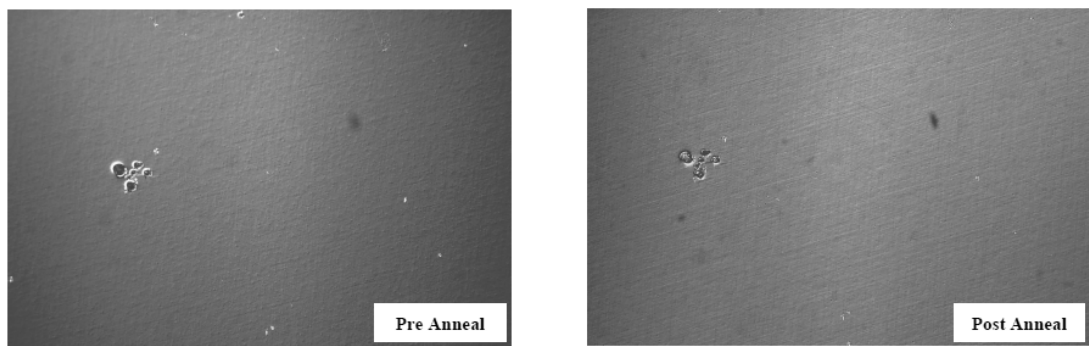

Figure 15. Optical microscopy images of the $\mathrm{HgCdTe/Si} \mathrm{before} \mathrm{and} \mathrm{after} \mathrm{ex-situ} \mathrm{cycle} \mathrm{annealing.} \mathrm{Note} \mathrm{the} \mathrm{landmark}$ defect used to identify the same area of material for both pre and post anneal images [59].

\begin{tabular}{|c|c|c|c|}
\hline \multicolumn{4}{|c|}{ Substrate Technology } \\
\hline & Bulk CdZnTe & $\mathrm{Si}$ & Advantage \\
\hline Maximum size & $7 \times 7 \mathrm{~cm}^{2}$ & 6 inch diameter & $\mathrm{Si}$ \\
\hline Maximum area & $\sim 50 \mathrm{~cm}^{2}$ & $\sim 180 \mathrm{~cm}^{2}$ & $\mathrm{Si}$ \\
\hline Scalability & No & Yes & $\mathrm{Si}$ \\
\hline Cost & $\$ 220 / \mathrm{cm}^{2}$ & $\sim \$ 1 / \mathrm{cm}^{2}$ & $\mathrm{Si}$ \\
\hline Thermal match to Si ROIC & No & Yes & $\mathrm{Si}$ \\
\hline Robustness & Brittle & Hard & $\mathrm{Si}$ \\
\hline Lattice match to MCT & Yes & No & $\mathrm{CZT}$ \\
\hline Surface & Smooth & Smooth & None \\
\hline Orientation available & $(112)$ & $(112)$ & None \\
\hline Vendors & 1 (foreign) & Numerous (domestic) & $\mathrm{Si}$ \\
\hline Substrate quality (dislocations) & $<10000 \mathrm{~cm}^{2}$ & $<100 \mathrm{~cm}^{2}$ & $\mathrm{Si}$ \\
\hline Impurities & Low & Extremely low & $\mathrm{Si}$ \\
\hline
\end{tabular}

Table 1. Advantages of Si-based composite substrate technology for HgCdTe material development [59].

Table 1 highlights the advantages for transitioning away from a bulk CdZnTe substrate technology for large area HgCdTe IR detectors and focal plane arrays. Past effort has focused on using a Si-based composite substrate technology, specificallyCdTe/Si, for $\mathrm{HgCdTe}$ material development. As shown in Table 1, Si has better attributes with respect to bulk CdZnTe in every category except for lattice and thermal matching to $\mathrm{HgCdTe}$. The lattice mismatch between $\mathrm{Si}$ and $\mathrm{HgCdTe}$ is $19.3 \%$ and has proven to be a significant challenge to overcome. To address this issue, a great effort within the $\mathrm{HgCdTe}$ community has been expended on developing $\mathrm{MBE}$ grown $\mathrm{CdTe} / \mathrm{Si}$ as a composite substrate for subsequent $\mathrm{HgCdTe}$ growth. Much research and investigation has gone into understanding and improving the surface passivation, nucleation, buffer layer growth, and material characterization of CdTe/Si material itself. Currently, $\mathrm{CdTe}(112) / \mathrm{Si}(112)$ is of extreme high quality with x-ray rocking curve full width at half maximum (FWHM) values measuring less than 60 arcsec for an $8 \mu \mathrm{m}$ thick epilayer [59]. Significant efforts are also being expended in developing $\mathrm{HgCdTe}$ on $\mathrm{GaAs}$ substrates. 
Both composite substrate development, whether it is using $\mathrm{Si}$, GaAs or some other alternative substrate system, and $\mathrm{HgCdTe}$ material improvement are active areas of study within the Infrared community. In fact, it might be a combination of techniques currently being developed that ultimately lead to $\mathrm{HgCdTe}$ grown on scalable alternative substrates supplanting $\mathrm{HgCdTe}$ grown on bulk CdZnTe substrates for large area array applications [60].

Figure 16 shows a $\mathrm{HgCdTe} / \mathrm{Si}$ FPA architecture hybridized via indium interconnects to the silicon Readout Integrated Circuit (ROIC). The FPA in Figure 16 consists of MBE grown $\mathrm{HgCdTe}$ single band detector arrays with in-situ doped, P-on-N architecture fabricated on a 6-inch silicon substrate.

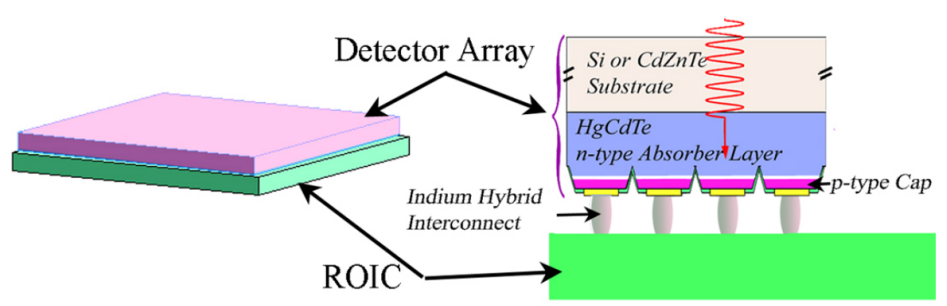

Figure 16. Architecture of an IRFPAs made with HgCdTe/Si hybridized to a Read Out Integrated Circuit (ROIC) [61].

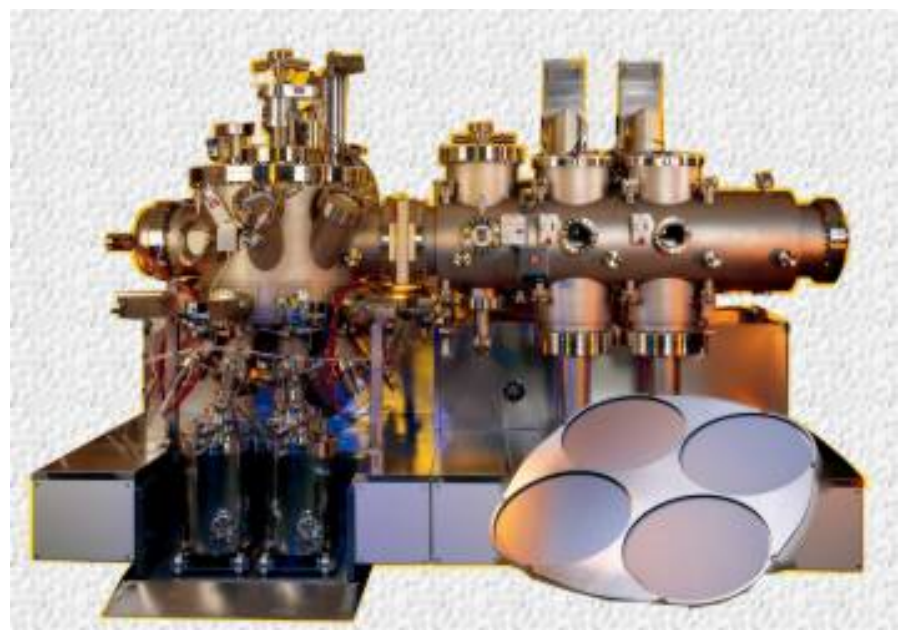

Figure 17. VG V100 Molecular Beam Epitaxial System

Using MBE system (see Figure 17), researchers produced epitaxial HgCdTe layers on (211) Si substrates with very low macro defect density and uniform Cd composition across the epitaxial wafers. These $\mathrm{HgCdTe} / \mathrm{Si}$ composite wafers have shown growth defect densities less than 10 defects $/ \mathrm{cm}^{2}$, approximately 100 times better than can be achieved on CdZnTe substrates, due to the better crystalline quality of the starting substrate [61]. 
The HgCdTe/Si epitaxial substrates with a P-on-N configuration can be fabricated into mesa delineated detectors using the same etch, passivation, and metallization schemes as detectors processed on $\mathrm{HgCdTe} / \mathrm{CdZnTe}$ substrates. Detector fabrication processes across the full area of 6-inch $\mathrm{HgCdTe} / \mathrm{Si}$ wafers have routinely produced high performing detector pixels from edge to edge of the photolithographic limits across the wafer, offering 5 times the printable area as compared with $6 \times 6 \mathrm{~cm}$ CdZnTe substrates. Large-format $(2 \mathrm{~K} \times 2 \mathrm{~K}) \mathrm{MWIR}$ FPAs fabricated using large area HgCdTe layers grown on 6-inch diameter (211) silicon substrates demonstrated NEDT operabilities better than 99.9\% (see Figure 18). SWIR and MWIR detector performance characteristic son $\mathrm{HgCdTe} / \mathrm{Si}$ substratesare comparable to those on the established $\mathrm{HgCdTe} / \mathrm{CdZnTe}$ wafers. HgCdTe devices fabricated on both types of substrates have demonstrated very low dark current, high quantum efficiency and full spectral band fill factor characteristic of $\mathrm{HgCdTe}$ [61].
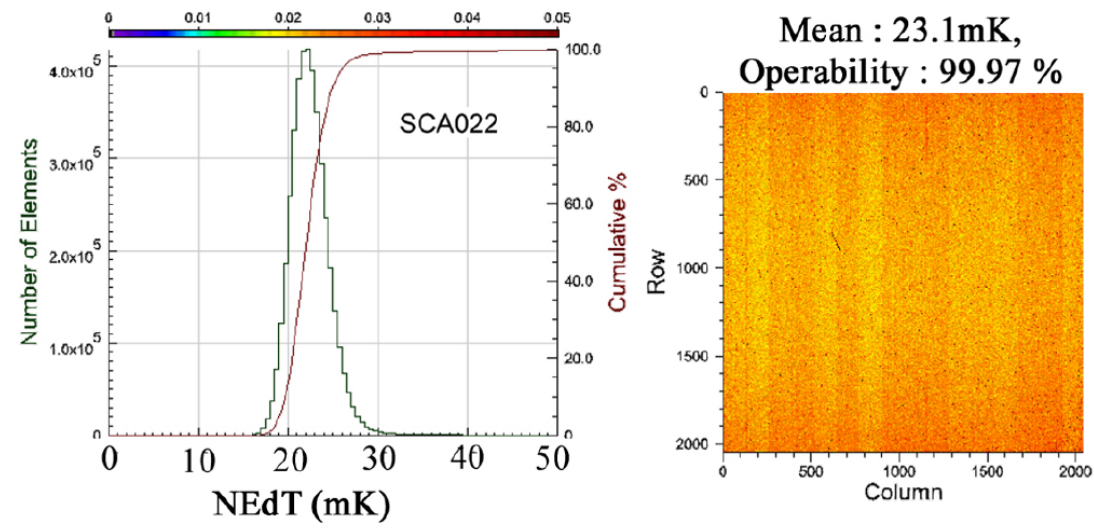

Figure 18. NEDT measured from $2 \mathrm{~K} \times 2 \mathrm{~K} \mathrm{HgCdTe} / \mathrm{Si} \mathrm{MWIR} \mathrm{HgCdTe} / \mathrm{Si}$ FPA with 15 micron unit cell demonstrating better than $99.9 \%$ operability [61].

\subsubsection{HgCdTe on Silicon Two-Color IRFPAs}

As noted above, the motivation for $\mathrm{HgCdTe}$ growth on large-area Si substrates is to enable larger array formats and potentially reduced FPA cost compared to smaller, more expensive $\mathrm{CdZnTe}$ substrates. In addition to the successful demonstration of single color IRFPA on composite $\mathrm{HgCdTe} / \mathrm{Si}$ substrates, researchers produced MWIR/LWIR dual band FPAs on large area Si substrates. The device structure is based on a triple-layer N-P-N heterojunction (TLHJ) architecture grown by molecular-beam epitaxy (MBE) on $100 \mathrm{~mm}$ (211) Si wafers with ZnTe and CdTe buffer layers [62]. The MWIR/LWIR dual band epitaxial wafers have low macro defect densities $\left(<300 \mathrm{~cm}^{-2}\right)$. Inductively coupled plasma etched detector arrays with 640x480 dual band pixels $(20 \mu \mathrm{m})$ are mated to dual-band readout integrated circuits (ROICs) to produce FPAs (see Figure 19). The measured $80 \mathrm{~K}$ cutoff wavelengths are $5.5 \mu \mathrm{m}$ for MWIR and $9.4 \mu \mathrm{m}$ for LWIR, respectively. The FPAs exhibit high pixel operabilities in 
each band, with noise equivalent differential temperature (NEDT) operabilities of $99.98 \%$ for the MWIR band and $99.6 \%$ for the LWIR band at $84 \mathrm{~K}$ [62].
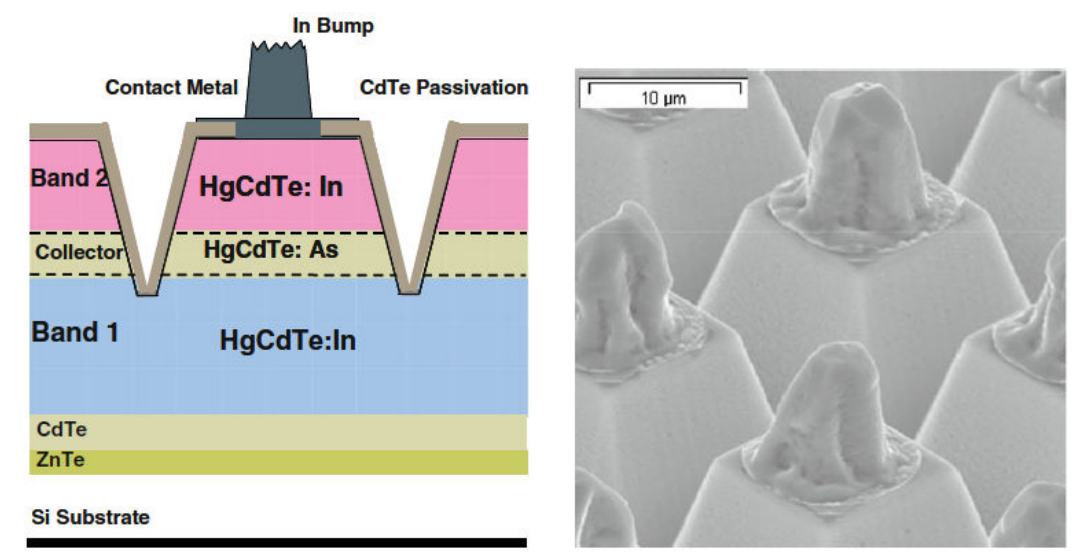

Figure 19. Cross-section of single-mesa dual-band detector architecture applied to HgCdTe on Si (Left). Shown on the Right is Scanning electron micrograph of 20-micron-unit-cell dual-band detectors array [62].

\subsection{High Operating Temperature (HOT) Detectors}

High performance MWIRand LWIR FPAs are normally cooled to cryogenic temperatures at about $80 \mathrm{~K}$, in order to suppress the dark current noise from overwhelming the photogenerated signals. If the operating temperature of the FPA is increased without degrading image quality, then smaller coolers can be used and the SWaP and cost of the system could be reduced. Of course, a more significant advantage can result if the operating temperature is increased to $>200 \mathrm{~K}$ where a low cost thermoelectric cooler can be implemented. There is a growing effort to increase the operating temperature of MWIR and LWIR infrared detectors by: reducing leakage currents; reducing thermal generation rates in the active region and minimizing the active volume of the detector without reducing quantum efficiency. While a number of strategies can be used to achieve high operating temperature (HOT) detectors, arecent DARPA program (AWARE-Broadband) focused on reducing detector material volume via a photon trap/photonic crystal approach to reduce dark current without degrading quantum efficiency [63-64].

The principle of volume reduction is demonstrated in Figure 20 which illustrates the effect of reducing the fill factor on device performance for a baseline shrinking mesa and an idealized photon trap detector. The fill factor is defined as the volume of material remaining divided by the volume of the unit cell. The mesa reduction in volume initially reduces the NEDT as noise generating volume is removed, until the volume removed causes the signal to be reduced relative to the noise. Two types of IR photon trapping structures have been investigated: In AsSb pyramidal arrays and $\mathrm{HgCdTe}$ pillars and holes. Photon trap detectors on MBE HgCdTe/Si epitaxial wafers (see Figure 21) exhibit improved performance com- 
pared to single mesas, with measured NEDT of $40 \mathrm{mK}$ and $100 \mathrm{mK}$ at temperatures of $180 \mathrm{~K}$ and $200 \mathrm{~K}$, with good operability. Large format arrays of these detectors exhibit cut-offs from $4.3 \mu \mathrm{m}$ to $5.1 \mu \mathrm{m}$ at $200 \mathrm{~K}$. For the In AsSb pyramidal arrays, the measured dark current at the bias for peaked QE is in the low $10^{-3} \mathrm{~A} / \mathrm{cm}^{2}$ range at $200 \mathrm{~K}$ and low $10^{-5} \mathrm{~A} / \mathrm{cm}^{2}$ range at $150 \mathrm{~K}$ [64]. The general $\mathrm{nBn}$ band diagram and the dark current density curves at various temperatures are shown in Figure 22a and b, respectively. The I-V curves shown in figure $22 \mathrm{~b}$ are from anCBn design, where the $\mathrm{C}$ stands for compound.

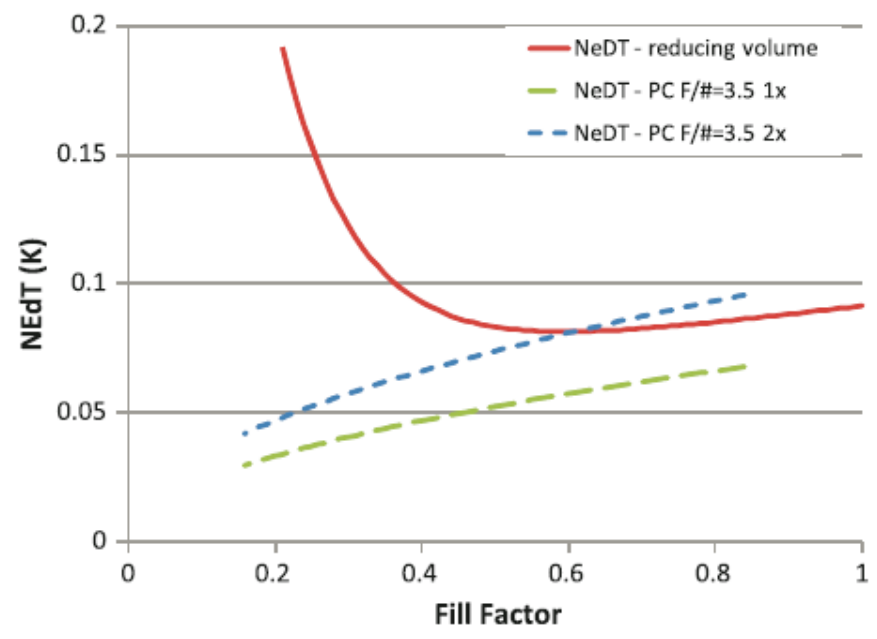

Figure 20. NEDT for a HgCdTe detector with a $5 \mu \mathrm{m}$ cutoff at $200 \mathrm{~K}$ for baseline reducing mesa approach compared with a photonic crystal approach for $1 x$ mean NEDT operability metric and 2x mean NEDT operability metric [63].

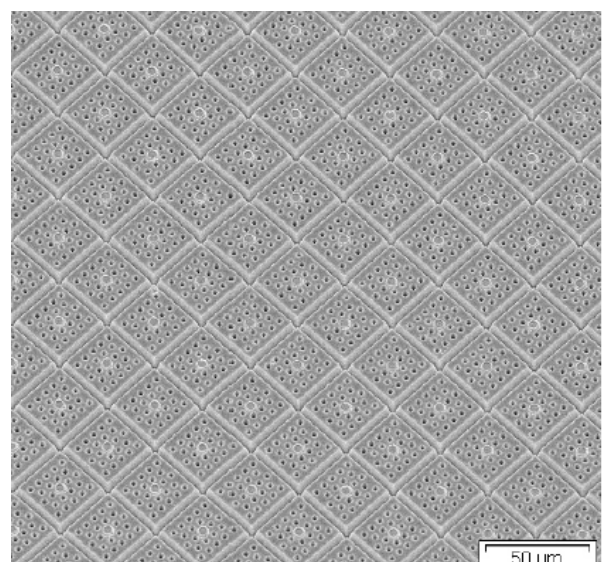

Figure 21. x $51230 \mu \mathrm{m}$ array consisting of unit cell design with photonic crystal holes on a $5 \mu \mathrm{m}$ pitch in a MWIR HgCdTe [63]. 


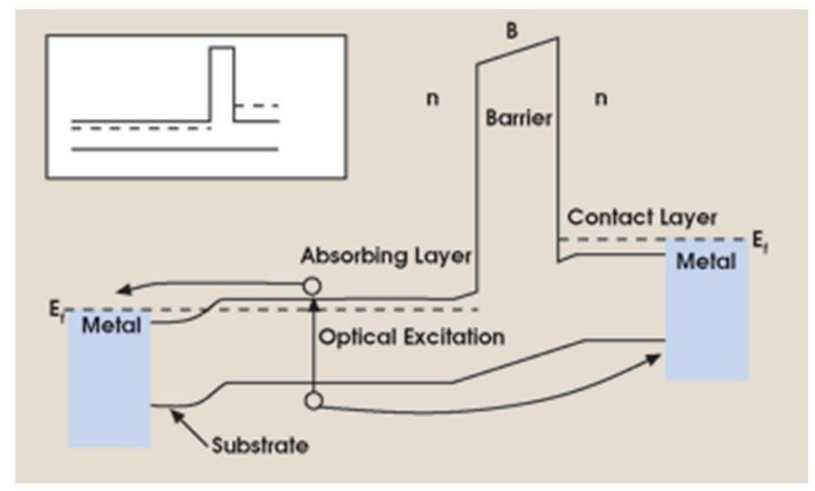

(a)

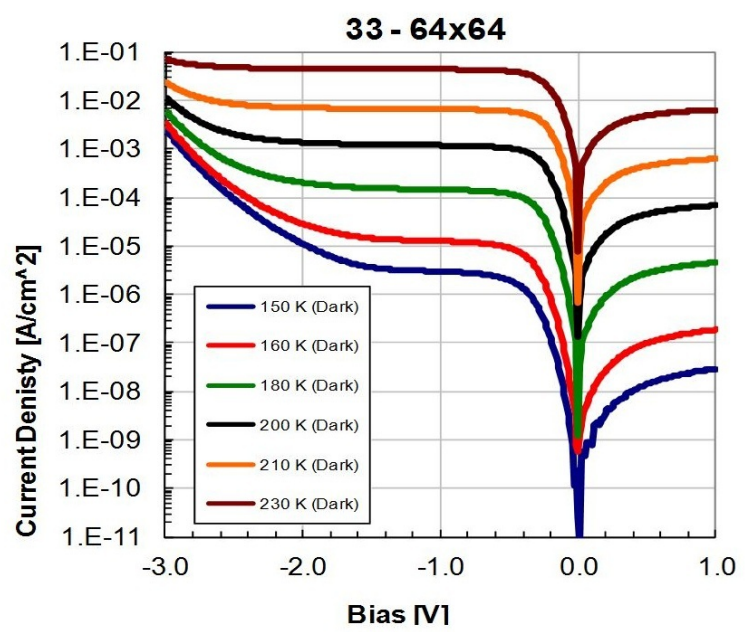

(b)

Figure 22. a) nBn band diagram illustrating the carrier flow [http://www.photonics.com/Article.aspx?AID=27744]. b) Dark current density curves as a function of temperature for an $\mathrm{nCBn}$ pyramidal $\ln A s_{1-x} \mathrm{Sb}_{\mathrm{x}}$ detector with a cutoff of $5.05 \mu \mathrm{m}$ at $200 \mathrm{~K}[64]$.

The nBn [65] detector design consists of a n-type absorption layer, a conduction band offset barrier layer and a n-type contact layer. This design suppresses majority carrier currents (electrons in this case) while maintaining low electric fields. The ideal design would require a flat valence band as shown in the inset of figure 22(a). However, in practice and depending on the choice of materials used, a small valence band offset may or may not exist. The conduction band large potential barrier blocks the flow of electrons while the flat valence band allows easy flow of holes. As a result, the thermally generated majority carrier, which contributes to dark current, is suppressed. Because the nBn architectures suppress the thermal noise, it is very suitable to operate this device at higher temperatures. As mentioned above (also see figure 22(b)), operation as high as $150 \mathrm{~K}$ with excellent performances have been demonstrated under the DARPA AWARE program. 


\subsection{Type II Strained Layer Superlattices (T2SL)}

Proposed by Smith and Mailhiot [66] in 1987, detectors based on InAs/GaSb strained layer superlattice (SLS) have attracted a lot of attention over the past few years as a possible alternative to the II-VI based IR sensors. The motivation for pursuing the III-V based SLS resulted from two major difficulties with LWIR MCT detectors: large tunneling currents and precise compositional control for accurate cutoff wavelengths. The InAs/GaSb SLS is engineered to achieve small bandgap materials with thin repeating layers for enhanced optical absorption and good electrical transport in the growth direction. The SLS structure typically consists of alternating layers with thicknesses varying from 4-20 nm.

These InAs/GaSb heterostructures are characterized by the broken-gap type-II alignment where the conduction band of the InAs layer is lower than the valence band of the GaSb layer as illustrated in Figure 23. The bandgap is the energy difference between the top of the heavy-hole mini-band (HH1) and the bottom of the electron miniband (C1), as indicated in Figure 23. The overlap of electron (hole) wave functions between adjacent InAs (GaSb) layers results in the formation of an electron (hole) minibands in the conduction (valence) band. For IR sensing, optical transitions between holes localized in GaSb layers and electrons confined in InAs layers are employed. As the layer thickness decreases, the wave-function overlap increases causing a more favorable optical absorption. As the thickness is increased beyond about $5 \mathrm{~nm}$, the wavefunction overlap is reduced with a corresponding decrease in optical coupling. The effective bandgap of the InAs/InGaSb SLs can be tailored from 3 um to 30 um abroption by varying the thickness of the constituent layers, thus enabling detectors spanning the entire IR spectrum [67-68].

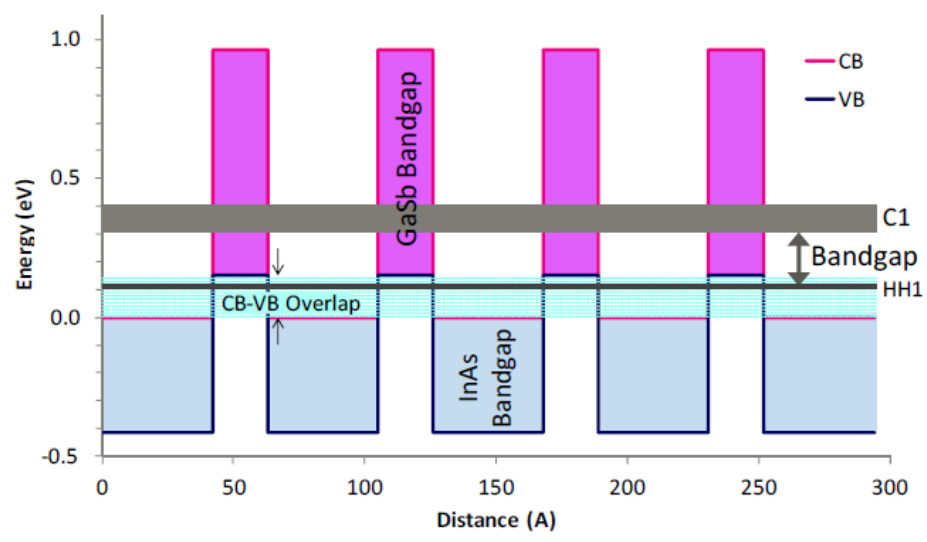

Figure 23. Schematic bandgap alignment of Type II InAs/GaSb superlattices [69].

The effective mass of the charge carriers in the superlattice is not dependent on the semiconductor bandgap, as in the case of bulk materials. The larger effective mass of the electrons and holes in SLs combined with the slower Auger recombination rate can lead to a reduc- 
tion of tunneling currents and higher operating temperatures compared to HgCdTe. The large splitting between heavy-hole and light-hole valence sub-bands, due to strain in the SLs, contributes to the suppression of Auger recombination rate. The maturity of the III-V materials technology offers technological advantages to the SLS effort by providing a source of commercially available low defect density substrates and recipes for very uniform processes utilizing large area substrates. This makes detectors based on SLs an attractive technology for realization of high performance single element detectors and FPAs [67-68].

SLS detectors are fabricated with either a p-on-n or n-on-p photodiode design. In either case, the optically active area of the photodiode is defined by an etched mesa as shown in Figure 24 (a). During the mesa isolation process, the periodic nature of the idealized crystal structure ends abruptly at the mesa sidewall surface. Disturbance of the periodic potential function, due to a broken crystal lattice, leads to allowed electronic quantum states within the energy band gap of the SLS resulting in large surface leakage currents. The suppression of these currents is the most demanding challenge for present day SLS technology, especially for LWIR and VLWIR spectral regions, since the dimensions of the SLS pixels have to be scaled to about $20 \mu \mathrm{m}$. The limitation imposed by surface leakage currents can be avoided by depositing a stable surface passivation layer onto the mesa sidewall. Currently, there is a lack of a robust passivating material and approach. The proposed approaches include: deposition of polyimide layer, overgrowth of wide band gap material, deposition of passivation sulphur coating electrochemically and post etch treatment in chemical solutions. However, these methods can affect cut-off wavelength of the device or complicate the fabrication process of the detectors [67-68].

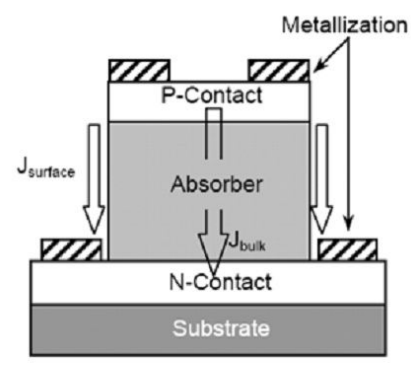

(a)

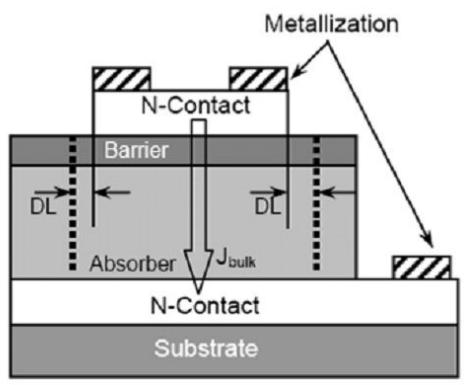

(b)

Figure 24. Schematic of (a) conventionally defined mesa (b) shallow etched isolation nBn device. In the latter case, the area is defined by the by diffusion length (DL) of the minority carriers (holes) [68].

A new heterostructure design to limit the surface leakage currents is depicted in Figure 24 (b) as the $\mathrm{nBn}$ architecture. Similar to the nBn discussion above, the SLS-nBn design allows for flexibility in employing band-engineered structures. The nBn detector consists of an n-type narrow band-gap contact that is separated from the absorber layers by a 50-100 nm thick, wide band-gap barrier layer. Unlike a conventional photodiode fabrication, the size of the nBn de- 
vice is defined by the lateral diffusion length of minority carriers (holes), as illustrated in Figure 24 (b). A $100 \mathrm{~K}$ increase in the BLIP temperature has been demonstrated [68]. SLS-based detectors with nBn design and special processing schemes showed dark current reduction of two orders of magnitude (at $77 \mathrm{~K}$ ) in comparison to conventional photodiode processing techniques. Quantum efficiency and shot-noise-limited specific detectivity are comparable to current SLS-based p-i-n diodes. While nBn detectors have been demonstrated, focal plane arrays based on InAs/GaSb SLS detectors with nBn designs are being developed [68-69].

\subsection{Microbolometers}

A microbolometer is a resistive element fabricated from a material that has a very small thermal capacity and a large temperature coefficient of resistance (TCR). The absorbed IR radiation is converted into heat which changes the resistance of the microbolometer such that measurable electrical signals can be detected.The spectral response of the bolometer is flat across the IR spectrum, since the sensing mechanism isindependent of the photoexcited carriers jumping across an energy band-gap. A schematic representation of a bolometer pixel on top of a ROIC is shown in Figure 25 details of which can be found in reference 70 . The pixel design shows a resonant cavity formed by an absorbing layer suspended above a reflecting metal layer. The cavity is used to amplify the absorptance of the incident IR radiation. The microbridge is supported by two beams and is thermally isolated from the ROIC to increase the sensitivity of the microbolometer.
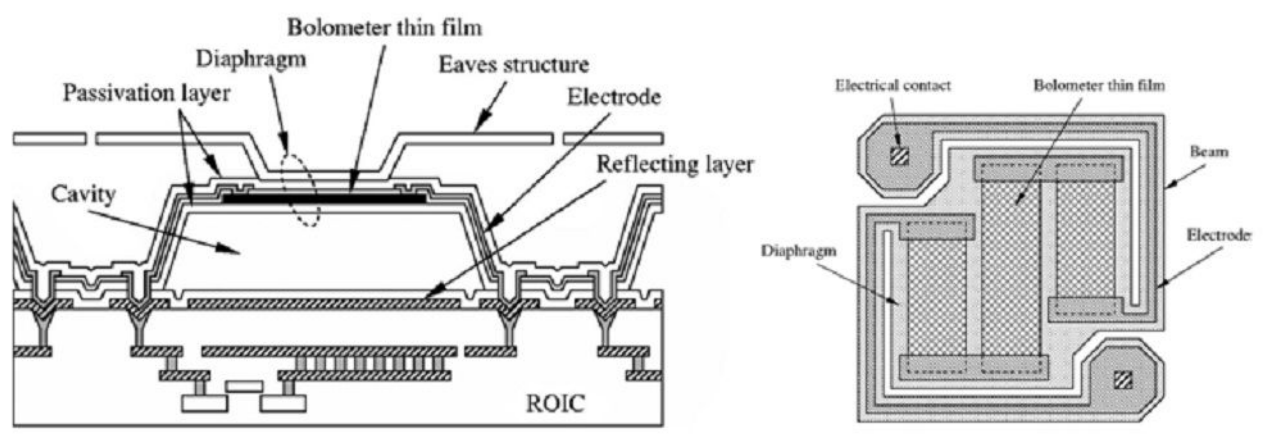

Figure 25. Schematic cross sectional and top views of Microbolometer IR Detector [70].

The microbolometer based on Si-MEMS device structure has been under development for over 20 years with support from DARPA and the Army. Most microbolometer structures utilize $\mathrm{VO}_{\mathrm{x}}$ and amorphous silicon thin film technologies. Companies such as Raytheon, BAE Systems and DRS Technologies are developing and producing 17 micron pixels in 640x 480 and larger arrays using $\mathrm{VO}_{x}$ [71-72]. L3 Communications and CEA-LETI are developing and producing $640 \times 480$ arrays with 17 micron unit cells using amorphous-Silicon technology [73-74]. Figure 26 presents examples of the microbolometer structures using $\mathrm{VO}_{\mathrm{x}}$ and amorphous silicon technologies. 


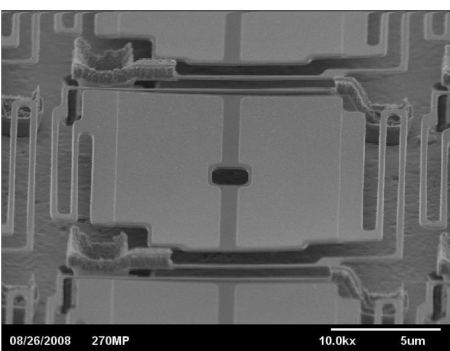

(a)

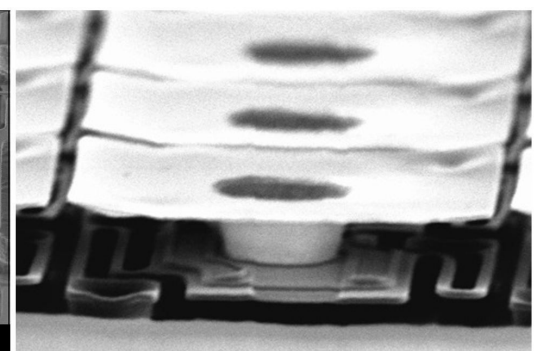

(b)

Figure 26. SEM images of a) amorphous Si and b) VO microbolometers IR Detector [72-73].

\section{Future IR Technology Directions}

Further advancement in imaging systems requires solutions for many fundamental and technological issues related to wide field of view (FOV), resolution, pixel pitch, optics, multicolor, form-factor, low SWaP, and low cost. This section highlights two projects currently fielded through the Microsystems Technology Office (MTO) of the Defense Advanced Project Agency (DARPA) to prepare for these advancements. The first is called AWARE (Advanced Wide Field of View Architectures for Image Reconstruction and Exploitation) and addresses several fundamental issues that will enable technologies for wide field-ofview, pixel scaling, broadband and multiband imaging [75-77]. The second project is LCTI$\mathrm{M}$ (Low Cost Thermal Imager-Manufacturing) which is addressing a cost effective solution for manufacturing microbolometers.

Traditional detector arrays are typically designed for a narrow band of wavelengths due to inadequate absorption and charge collection from photons with varying wavelengths. Broadband absorption is usually inadequate due to quantum efficiency (QE) roll-off. To design a detector with high $\mathrm{QE}$, low NETD and high operating temperature across a very broad band of wavelengths, say visible to 5 um range, traditional detector design would be less than optimum. A detector design that can accomplish these goals is based on a photonic pillar-type architecture. Photonic crystals are relatively well understood and have been demonstrated for applications like VCSELs, which are similar to photovoltaic detectors. Sub-wavelengthsize semiconductor pillar arrays within a single detector can be designed and structured as an ensemble of photon trapping units to significantly increase absorption and QE for a wide band of wavelengths. Each sub-element in each pixel can be a 3D photonic structure fabricated using either a top-down or bottom-up process scheme. The sub-element architecture canbe of different shapes such as pyramidal, sinusoidal or rectangular [75].

Using unique pyramidal and pillar topologies etched into the photon absorbing layer, researchers have demonstrated 3D photon trapping, achieved significant reduction in dark current and established uniform QE (see Figure 27). This is the first demonstration of broad- 
band performance in a single infrared detector using a pillared microstructure in a semiconducting material. The broadband technology has been demonstrated independently in II-VI and III-V based epitaxial materials. This achievement paves the way to replace multiple cameras with one [75]. It also gives the ability for hyper spectral sensing that will enable better target discrimination compared to a single narrow band camera. The high performance at $200 \mathrm{~K}$ compared to traditional $80 \mathrm{~K}$ operation allows for a smaller SWaP design, since high power and large cryogenic coolers can be replaced by low power miniature coolers. Such cameras would have significant impact on smaller platforms.
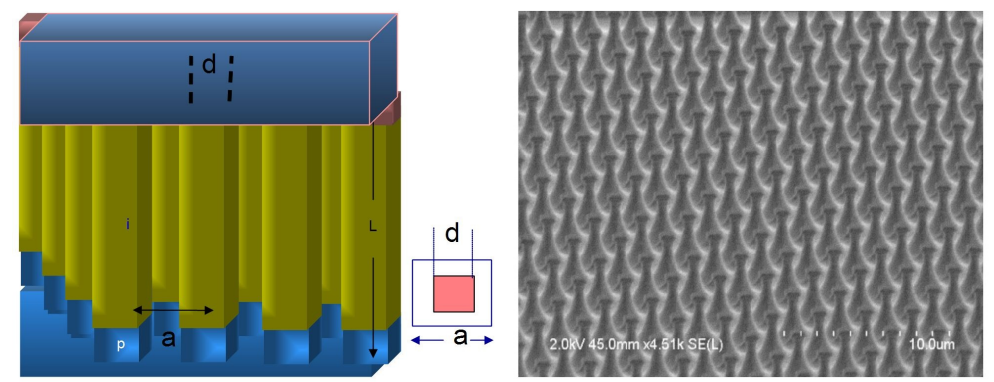

Figure 27. (Left) Illustration of pillared photonic detector architecture; (right) Micrograph of pyramidal PT structure fabricated in epitaxial InAsSb.

The pixel scaling effort is developing very high density LWIR and MWIR FPAs with pixel dimensions approaching the Nyquist- limit. Unlike visible sensors where the pixel size has been reduced to $1.4 \mathrm{um}$, the scaling of infrared pixels is much more difficult. As the pixel size is reduced, "bump-bonding", ROIC, signal integrating capacitor and signal to noise ratio become difficult. Achieving very small pixels however, will enable larger FPAs with small optics and cold shield, better resolution and yielding a huge reduction in SWaP. In one of the approaches, three layers (detector array, ROIC and MEMS capacitor array) are being developed separately, followed by integrating individual cells via indium bumps and through silicon vias (TSV).

To achieve high sensitivity (say $<30 \mathrm{mK}$ ) LWIR FPAs with $5 \mu \mathrm{m}$ pixels require large amounts of integrated charge to be accommodated in a very small unit cells. For a $5 \mu \mathrm{m}$ planar unit cell, the charge capacity in standard ROIC technology is less than 1 million electrons, whereas 8 to 12 million electrons are required for good sensitivity - a reason why small pitch IR detectors are not available today. As an enabler for this small pitch LWIR detector, the challenge of charge storage in small pixels is being addressed by fabricating MEMS capacitors suited to a 3D ROIC design. The MEMS capacitor array can be fabricated in a separate 8 " wafer. This technology yielded 20 million electrons in a 5 micron unit cell. This breakthrough will pave the way for small pitch FPAs to operate with very high sensitivity. Figure 28(b) shows a Transmission Electron Micrograph (TEM) picture of a portion of the MEMS capacitor array. Using the High Density Vertically Integrated Photodetector (HDVIP) technology, a fully functional 1280X720, $5 \mu \mathrm{m}$ unit cell LWIR FPA has been dem- 
onstrated [75]. The characterization shows excellent results with $99.8 \%$ response operability within $50 \%$ of the median.

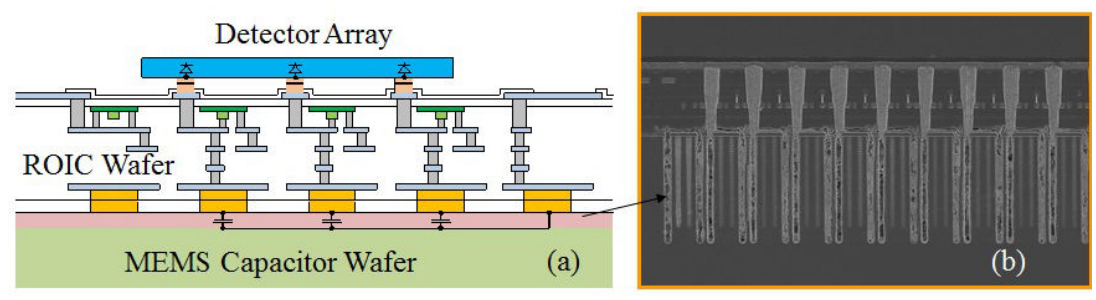

Figure 28. a) Schematic illustrationof the 3D integrated AWARE Lambda-Scale LWIR FPA design; b) Micrograph of the MEMS capacitor array cross section [75].

Another DARPA program, LCTI-M addresses the development of an advanced low cost room temperature IR cameras based upon cell phone CMOS camera technology, where the imaging sensor, optics and electronics are fabricated at the wafer level. IR imaging capability, such as thermal cameras, provides significant advantages in terms of visibility and target detection in all weather conditions making it a vital tool for day/night operations. However, the cost of thermal camerasis one of the key factors limiting the availability of high performance IR imagers at consumer level. Further, current form-factors are unacceptable for new applications in smaller handheld devices (such as PDAs) and glasses similar to Google Glass. Availability of very low cost and small form-factor IR cameras will enable a variety of applications such as fire-fighting, security, medical and gaming industry.

Nanotechnology and science of emerging materials and material designs have stirred up a slew of research that has significant impact on sensors and many other electronic devices. Nanostructures offer very thin absorption layers due to many intricate designs such as plasmonics and metamaterials to concentrate photons and enhance electric field. The advantages of thinner absorber in a photodetector means shorter carrier transit time, thus high speed detectors and greater structural compatibility with ever-shrinking electronic devices. Many advances in nanomaterials for detectors have been made allowing for very low cost technology. A detailed review of nano-based detector research is given in reference [78].

DARPA has funded several new approaches for detector development using carbon nanotube, graphene, nanoparticles and other nanomaterials. These researches demonstrate high potential for future detector technologies that could be very beneficial for both, military and commercial sectors.

\section{Summary}

In this chapter, we have discussed growth, fabrication and characteristics of mainstream infrared materials and devices on a variety of substrates. We have discussed SWIR band of interest 
that involves InGaAs, SiGe and HgCdTe based technologies and their applications. We also discussed the technologies and applications of MWIR, LWIR and multi-color devices for the 3-5 and 8-14 micron bands. Some of the key work discussed includes InSb, HgCdTe, and III-V based $\mathrm{nBn}$ and Strained Layer Super Lattice (SLS). Discussion of thermal bolometer devices provide introduction to future low cost LWIR technology. Each of these technologies has a place in the infrared band where a variety of detector configurations are being used. We also discussed the application of photonic type structures to IR detectors with broadband spectral response and high operating temperatures. It was shown that sub-wavelength size semiconductor pillar arrays can be designed and structured as an ensemble of photon trapping units to significantly increase absorption and QE over a wide band of wavelengths. It is anticipated that the current research and development presented in Section 7 will enable a host of new technologies for a variety of defense and commercial applications.

Although numerous research activities are ongoing in the area of nanoscience and technology, we briefly made comments on such technologies to make readers aware of various research activities.

\section{Acknowledgements}

The authors gratefully acknowledge the contributions of the many performers on the DARPA-AWARE program who have provided the results described in this manuscript and have been essential in advancing new infrared imaging capabilities.

\section{Author details}

Nibir K. Dhar ${ }^{1 *}$, Ravi Dat ${ }^{2}$ and Ashok K. Sood ${ }^{3}$

*Address all correspondence to:

1 Defense Advanced Project Agency, Arlington

2 Booz Allen Hamilton, Arlington, USA

3 Magnolia Optical Technologies Inc., Woburn, USA

\section{References}

[1] Pollehn, H., Choi, K. K., Svensson, S., \& Dhar, N. (2007). IR Material Research at the Army Research Laboratory," Invited Keynote Paper. Proceedings of SPIE, 6542C. 
[2] Martin, T. J., Cohen, M. J., Dries, J. C., \& Lange, M. J. (2004). InGaAs/InP Focal Plane Arrays for Visible Light Imaging. Proceedings of SPIE, 5406, 38-45.

[3] Davis, M., Devitt, J., Greiner, M., Rawe, R., Timlin, A., \& Wade, D. (2004). Advanced FPA Technology Development at CMC Electronics. Proceedings of SPIE, 5563, 62-73.

[4] Becker, L. (2005). Current and Future trends in Infrared Focal Plane Array technology" Invited Paper. Proceedings of SPIE, 5881, 105.

[5] Dereniak, E. L., \& Boreman, G. D. (1996). Infrared Detectors and Systems, Wiley, New York, NY.

[6] Kinch, M. A. (2007). Fundamentals of Infrared Detector Materials, SPIE Press, Bellingham, WA.

[7] Rogalski, A. (2011). Infrared detectors, CRC Press, Boca Raton, FL.

[8] Reine, M. B., Sood, A. K., \& Tredwell, T. J. (1981). Photovoltaic Infrared Detectors. in Semiconductors and Semimetals, eds. R. K. Willardson and A. C. Beer, 18, 201-311, Academic Press, New York.

[9] Micklethwaite, W. F. H. (1981). The Crystal Growth of Mercury Cadmium Telluride. Semiconductors and Semimetals, eds. R. K. Willardson and A. C. Beer, 18, 48-119, Academic Press, New York.

[10] Tregilgas, J. H. (1994). Developments in Recrystallized Bulk HgCdTe. Progress in Crystal Growth and Characterization of Materials, 28, 57-83.

[11] Triboulet, R. (1994). The Travelling Heater Method (THM) for Hg1-xCdxTe and Related Materials. Progress in Crystal Growth and Characterization of Materials, 28, 85-114.

[12] Capper, P. (2006). Bulk Crystal Growth- Methods and Materials. in Springer Handbook of Electronic and Photonic Materials, (Eds.) S. Kasap and P. Capper, Springer Science, New York.

[13] Capper, P. (1989). Bridgman Growth of CdxHg1-xTe: A Review. Progress in Crystal Growth and Characterization of Materials, 19, 259-93.

[14] Capper, P., Maxey, C., Butler, C., Grist, M., \& Price, J. (2004). Bulk growth of near-IR cadmium mercury telluride (CMT). Journal of Materials Science: Materials in Electronics, $15,721-725$.

[15] Colombo, L., Chang, R. R., Chang, C. J., \& Baird, B. A. (1988). Growth of Hg-Based Alloys by the Travelling Heater Method. Journal of Vacuum Science and Technology A, 6, 2795-99.

[16] Lanir, M., \& Riley, K. J. (1982). Performance of PV HgCdTe Arrays for 1-14- $\mu$ m Applications. IEEE Transactions on Electronic Devices ED, 29, 274-79.

[17] Wang, C. C. (1991). Mercury Cadmium Telluride Junctions Grown by Liquid Phase Epitaxy. Journal of Vacuum Science and Technology B, 9, 1740-45. 
[18] Pultz, G. N., Norton, P. W., Krueger, E. E., \& Reine, M. B. (1991). Growth and Characterization of p-on-n HgCdTe Liquid-Phase Epitaxy Heterojunction Material for 11-18 $\mu \mathrm{m}$ Applications. Journal of Vacuum Science and Technology B, 9, 1724-30.

[19] Tung, T., Kalisher, M. H., Stevens, A. P., \& Herning, P. E. (1987). Liquid-Phase Epitaxy of Hg1-xCdxTe from Hg Solution: A Route to Infrared Detector Structures. $\mathrm{Ma}$ terials Research Society Symposium Proceedings, 90, 321-56.

[20] Tung, T., De Armond, L. V., Herald, R. F., Herning, P. E., Kalisher, M. H., Olson, D. A., Risser, R. F., Stevens, A. P., \& Tighe, S. J. (1992). State of the Art of Hg-melt LPE HgCdTe at Santa Barbara Research Center. Proceedings of SPIE, 1735, 109-31.

[21] Herning, P. E. (1984). Experimental Determination of the Mercury-Rich Corner of the Hg-Cd-Te Phase Diagram. Journal of Electronic Materials, 13, 1-14.

[22] Harman, T. C. (1980). Liquidus Isotherms, Solidus Lines and LPE Growth in the TeRich Corner of the Hg-Cd-Te System. Journal of Electronic Materials, 9, 945-61.

[23] Tung, T., Su, C. H., Liao, P. K., \& Brebrick, R. F. (1982). Measurement and Analysis of the Phase Diagram and Thermodynamic Properties in the Hg-Cd-Te System. Journal of Vacuum Science and Technology, 21, 117-24.

[24] Bowers, J. E., Schmit, J. L., Speerschneider, C. J., \& Maciolek, R. B. (1980). Comparison of HgCdTe LPE Layer Growth from $\mathrm{Te}, \mathrm{Hg}$, and HgTe Rich Solutions. IEEE Transactions on Electron Devices, 27, 24-28.

[25] Schmit, J. (1983). Growth, Properties and Applications of HgCdTe. Journal of Crystal Growth, 65, 249-261.

[26] Chen, M. C., \& Dodge, J. A. (1986). Electrical properties of antimony-doped p-type $\mathrm{Hg} / \mathrm{sub} 0.78 / \mathrm{Cd} / \mathrm{sub} 0.22 /$ Te liquid-phase-epitaxy films. Solid State Communications, $59,449-452$.

[27] Baker, I. M. (2002). HgCdTe 2D Arrays-Technology and Performance Limitis in. Handbook of Infrared Detection Technologies, eds. M. Henini and M. Razeghi, Elsevier Science, New York, NY.

[28] Colombo, L., Westphal, G. H., Liao, P. K., Chen, M. C., \& Schaake, H. F. (1992). Infrared focal plane array producibility and related materials. Proceedings of SPIE, 1683, 33.

[29] Mitra, P., Case, F. C., \& Reine, M. B. (1998). Progress in MOVPE of HgCdTe for Advanced Infrared Detectors. J. Electron. Mater., 27(6), 510-520.

[30] Hipwood, L. G., Baker, I. M., Jones, C. L., Maxey, C., Lau, H. W., Fitzmaurice, J., Wilson, M., \& Knowles, P. (2008). LW IRFPAs made from HgCdTe grown by MOVPE for use in Multispectral Imaging. Proceedings of SPIE, 6940.

[31] Wu, O. K. (1993). Status of HgCdTe MBE technology for IRFPA. Proceedings of SPIE, 202179. 
[32] , J., \& Faurie, P. (1994). Molecular Beam Epitaxy of HgCdTe: Growth and Characterization. Prog. Crystal Growth and Charact, 29, 85-159.

[33] Dat, R., Aqariden, F., Duncan, W. M., Chandra, D., \& Shih, H. D. (1997). In Situ Spectroscopic Ellipsometry for Real Time Composition Control of HgCdTe Grown by Molecular Beam Epitaxy. Mater. Res. Soc. Symp. Proc., 484, 613-18.

[34] Irvine, S. I. C., \& Bajaj, J. (1994). Recent progress with in situ monitoring of MCTGrowth. Proceedings of SPIE, 2274, 24-36.

[35] Lee, T. S., Garland, J., Grein, C. H., Sumstine, M., Jandeska, A., Selamet, Y., \& Sivananthan, S. (2000). Correlation of Arsenic Incorporation and Its Electrical Activation in MBE HgCdTe. Journal of Electronic Materials, 29, 869-72.

[36] Tennant, W. E., Cockrum, C. A., Gilpin, J. B., Kinch, M. A., Reine, M. B., \& Ruth, R. P. (1992). Key Issue in HgCdTe-Based Focal Plane Arrays: An Industry Perspective. Journal of Vacuum Science and Technology B, 10, 1359-69.

[37] Triboulet, R., Tromson-Carli, A., Lorans, D., \& Nguyen Duy, T. (1993). Substrate Issues for the Growth of Mercury Cadmium Telluride. Journal of Electronic Materials, 22, 827-34.

[38] Gertner, E. R., Tennant, W. E., Blackwell, J. D., \& Rode, J. P. (1985). HgCdTe on sapphire: A New Approach to Infrared Detector Arrays. Journal of Crystal Growth, 72, 462-67.

[39] Edwall, D. D., Chen, J. S., Bajaj, J., \& Gertner, E. R. (1990). MOCVD HgCdTe/GaAs for IR Detectors. Semiconductor Science and Technology, 5, 221-224.

[40] Dhar, N. K., Wood, C. E. C., Gray, A., Wei, H. Y., Salamanca-Riba, L., \& Dinan, J. H. (1996). Heteroepitaxy of CdTe on $\{211\}$ Si using crystallized amorphous ZnTe templates. J. Vac. Sci. Technol. B, 14(3), 2366-70.

[41] Peterson, J. M., Franklin, J. A., Readdy, M., Johnson, S. M., Smith, E., Radford, W. A., \& Kasai, I. (2006). High-Quality Large-Area MBE HgCdTe/Si. Journal of Electronic Materials, 35, 1283-86.

[42] van der Hoogeveen, R. J. A. R. W. M., Spruijt, H. J., \& Goede, A. P. H. (1997). Low noise InGaAs infrared (1.0-2.4 microns) focal plane arrays for SCIAMACHY. Proc. SPIE, 2957, 54-65.

[43] Acton, D., Jack, M., \& Sessler, T. (2009). Large format short-wave infrared (SWIR) focal plane array (FPA) with extremely low noise and high dynamic range. Proceedings of SPIE, 7298, 72983E.

[44] Onat, B. M., Huang, W., Masaun, N., Lange, M., Ettenberg, M. H., \& Dries, C. (2007). Ultra low dark current InGaAs technology for focal plane arrays for low-light level visible-shortwave infrared imaging. Proceedings of SPIE, 6542, 65420L.

[45] Boisvert, J., Isshiki, T., Sudharsanan, R., Yuan, P., \& Mc Donald, P. (2008). Performance of very low dark current SWIR PIN arrays. Proceedings of SPIE, 6940, 69400L. 
[46] Mac, M., Dougal, J., Geske, J., Wang, , \& Follman, D. (2011). Short-wavelength infrared imaging using low dark current InGaAs detector arrays and vertical-cavity surface-emitting laser illuminators. Optical Engineering, 50(6), 061011.

[47] Yuan, H., Meixell, M., Zhang, J., Bey, P., Kimchi, J., \& Kilmer, L. C. (2012). Low Dark Current Small Pixel Large Format InGaAs 2-D Photodetector array Development. Proceedings of SPIE, 8353, 8353309.

[48] Kozlowski, L. J., Johnston, S. L., Mc Levige, W. V., Vanderwyck, A. H. B., Cooper, D. E., Cabelli, S. A., Blazejewski, E. R., Vural, K., \& Tennant, W. E. (1992). x128 PACE-I $\mathrm{HgCdTe}$ hybrid FPAs for thermoelectrically cooled applications. Proceedings of SPIE, 1685, 193-204.

[49] D’Souza, A. I., Robinson, E. W., Stapelbroek, M. G., Wong, W., Skokan, M., \& Shih, D. H. (2009). Visible to SWIR Response of HgCdTe HDVIP Detectors. Proceedings of SPIE, 7298, 72981X-1.

[50] Hansen, G. L., Schmit, J. L., \& Casselman, T. N. (1982). Energy gap versus alloy composition and temperature in $\mathrm{Hg}_{1-\mathrm{x}} \mathrm{Cd}_{\mathrm{x}}$ Te. J. Appl. Phys., 53, 7099-710.

[51] Gravrand, O., Mollard, L., Boulade, O., Moreau, V., Sanson, E., \& Destefanis, G. (2011). Ultra low dark current CdHgTe FPAs in the SWIR range at CEA and Sofradir. Proceedings of SPIE, 8176, $81761 \mathrm{H}$.

[52] Tsaur, B.-Y., Chen, C. K., \& Marino, S. A. (1991). Long-wavelength $\mathrm{Ge}_{x} \mathrm{Si}_{1-x} / \mathrm{Si}_{\text {hetero- }}$ junction infrared detectors and focal plane array. Proceedings of SPIE, 1540, 580-595.

[53] Wada, H., Nagashima, M., Hayashi, K., Nakanishi, J., Kimata, M., Kumada, N., \& Ito, S. (1999). 512x512 element GeSi/Si heterojunction infrared focal plane array. Proceedings of SPIE, 3698, 584-595.

[54] Sood, A. K., Richwine, R. A., Puri, Y. R., Di Lello, N., Hoyt, J. L., Akinwande, T. I., Horn, S., Balcerak, R. S., Bulman, G., \& Venkatasubramanian, R. (2009). Development of Low Dark Current SiGe-Detector Arrays for Visible-Near IR Imaging Sensor. Proceedings of SPIE, 7298, 72983D.

[55] Bublik, V. T., Gorelik, S. S., Zaitsev, A. A., \& Polyakov, A. Y. (1974). Calculation on the Binding Energy of Ge-Si Solid Solution. Phys. Status Solidi, 65, K79-84.

[56] Sood, A. K., Richwine, R. A., Puri, Y. R., Olubuyide, O. O., Di Lello, N., Hoyt, J. L., Akinwande, T., Balcerak, R. S., Horn, S., Bramhall, T. G., \& Radack, D. J. (2008). Design considerations for SiGe-based NIR Imaging Sensor. Proceedings of SPIE, 6940, 69400M.

[57] Markovitz, T., Pivnik, I., Calahorra, Z., Ilan, E., Hirsch, I., Zeierman, E., Eylon, M., Kahanov, E., Kogan, I., Fishler, N., Brumer, M., \& Lukomsky, I. (2011). Digital 640x512 InSb detector for high frame rate, high sensitivity and low power applications. Proceedings of SPIE, 8012, 80122Y. 
[58] Hirsch, L., Shkedy, L., Chen, D., Fishler, N., Hagbi, Y., Koifman, A., Openhaim, Y., Vaserman, I., Singer, M., \& Shtrichman, I. (2012). Hybrid Dual-Color MWIR Detector for Airborne Missile Warning Systems. Proceedings of SPIE, 8353, 83530H.

[59] Brill, G., Chen, Y., Wijewarnasuriya, P., \& Dhar, N. K. (2009). Infrared Focal Plane Array Technology Utilizing HgCdTe / Si: Successes, Roadblocks and material Improvements. Proceedings of SPIE, 7419, 74190L.

[60] Chen, Y., Farrell, S., Brill, G., Wijewarnasuriya, P., \& Dhar, N. K. (2008). Infrared Focal Plane Array Technology using HgCdTe/Si Material Growth. Journal of Crystal Growth, 310(24).

[61] Bangs, J., Langell, M., Reddy, M., Melkonian, L., Johnson, S., Elizondo, L., Rybnicek, K., Norton, E., Jaworski, F., Asbrock, J., \& Baur, S. (2011). Large Format High Operability SWIR and MWIR Focal Plane Array Performance and Capabilities. Proceedings of SPIE, 8012, 801234.

[62] Patten, E. A., Goetz, P. M., Viela, F. A., Olsson, K., Lofgrren, D. F., Vodicka, J. G., \& Johnson, S. M. (2010). High-Performance MWIR/LWIR Dual-Band 640x480 HgCdTe/Si FPA's. Journal of Electronic Materials, 39(10).

[63] Smith, K. D., Wehner, J. G. A., Graham, R. W., Randolph, J. E., Ramirez, A. M., Venzor, G. M., Olsson, K., Vilela, M. F., \& Smith, E. P. G. (2012). High Operating temperature Mid-wavelength Infrared $\mathrm{HgCdTe}$ photon trapping focal plane arrays. Proceedings of SPIE, 8353, 83532R.

[64] D'Souza, A. I., Robinson, E., Ionescu, A. C., Okerlund, D., de Lyon, T. J., Rajavel, R. D., Sharifi, H., Yap, D., Dhar, N., Wijewarnasuriya, P. S., \& Grein, C. (2012). MWIR InAs1-xSbx nCBn Detectors Data and Analysis. Proceedings of SPIE, 8353, 835333.

[65] Maimon, S., \& Wicks, G. W. (2006). nBn detector, an infrared detector with reduced dark current and higher operating temperature. APL 89, 151109.

[66] Smith, D. L., \& Mailhot, C. (1987). Proposal for Strained type II superlattice infrared detectors. Journal of Applied. Physics, 62(2545).

[67] Rodriguez, J. B., Plis, E., Bishop, G., Sharma, Y. D., Kim, H., Dawson, L. R., \& Krishna, S. (2007). nBn structure based on InAs/GaSb type-II strained layer superlattices. Applied Physics Letters,, 91, 043514.

[68] Krishna, S. (2009). Infrared focal plane arrays based on dots in a well and strained layer superlattices. Proceedings of SPIE, 7222, 72220P.

[69] Ariyawansa, G., Duran, J. M., Grupen, M., Scheihing, J. E., Nelson, T. R., \& Eismann, M. T. (2012). Multispectral Imaging with Type II Superlattice detectors. Proceedings of SPIE, 8353, 835530E.

[70] Tohyama, S., Miyoshi, M., Kurashina, S., Ito, N., Sasaki, T., Ajisawa, A., Tanaka, Y., Kawahara, A., Iida, K., \& Oda, N. (2006). New thermally isolated pixel structure for 
high-resolution $(640 \times 480)$ uncooled infrared focal plane arrays. Optical Engineering, $45,014001$.

[71] Blackwell, R., Lacroix, D., \& Bach, T. (2009). micron microbolometer FPA Technology at BAE Systems. Proceedings of SPIE, 7298, 72980P.

[72] Li, C., Skidmore, G., Howard, C., Clarke, E., \& Han, J. (2009). Advancement in 17 micron pixel pitch uncooled focal plane arrays. Proceedings of SPIE, 7298, 72980 S.

[73] Schimert, T., Hanson, C., \& Brady, J. (2009). Advanced in small-pixel, large-format alpha-silicon bolometer arrays. Proceedings of SPIE, 7298, 72980T.

[74] Trouilleau, C., Fieque, B., Noblet, S., \& Giner, F. (2009). High Performance uncooledamorphous silicon TEC less XGA IRFPA with 17 micron pixel. Proceedings of SPIE, 7298, 72980Q.

[75] Dhar, N. K., \& Dat, R. (2012). Advanced Imaging Research and Development at DARPA. Proceedings of SPIE, 8353, 835302.

[76] D'Souza, A. I., Robinson, E., Ionescu, A. C., Okerlund, D., de Lyon, T. J., Rajavel, R. D., Sharifi, H., Yap, D., Dhar, N., Wijewarnasuriya, P. S., \& Grein, C. (2012). MWIR InAs1-xSbx nCBn Detectors Data and Analysis. Proceedings of SPIE, 8353, 835333.

[77] Smith, K. D., Wehner, J. G. A., Graham, R. W., Randolph, J. E., Ramirez, A. M., Venzor, G. M., Olsson, K., Vilela, M. F., \& Smith, E. P. G. (2012). High Operating temperature Mid-wavelength Infrared $\mathrm{HgCdTe}$ photon trapping focal plane arrays. Proceedings of SPIE, 8353, 8353 2R.

[78] Logeeswaran, V. J., Jinyong, O., Nayak, A. P., Katzenmeyer, A. M., Gilchrist, K. H., Grego, S., Kobayashi, N. P., Wang, S. Y., Talin, A. A., Dhar, N. K., \& Islam, M. S. (2011). A perspective on Nanowire Photodetectors: Current Status, Future Challenges, and Opportunities. IEEE Journal of selected topics in quantum electronics, 17(4), 1002-1023. 